\title{
Chronic B2AR stimulation limits CFTR activation in human airway epithelia
}

\author{
John J. Brewington, ${ }^{1}$ Jessica Backstrom, ${ }^{1}$ Amanda Feldman, ${ }^{1}$ Elizabeth L. Kramer, ${ }^{1}$ \\ Jessica D. Moncivaiz, ${ }^{1}$ Alicia J. Ostmann, ${ }^{1}$ Xiaoting Zhu, ${ }^{2}$ L. Jason Lu, ${ }^{2}$ and John P. Clancy \\ 'Division of Pulmonary Medicine, Department of Pediatrics, and 2Division of Biomedical Informatics, Department of \\ Pediatrics, Cincinnati Children's Hospital Medical Center, Cincinnati, Ohio, USA.
}

\begin{abstract}
Traditional pulmonary therapies for cystic fibrosis (CF) target the downstream effects of CF transmembrane conductance regulator (CFTR) dysfunction (the cause of CF). Use of one such therapy, $\boldsymbol{\beta}$-adrenergic bronchodilators (such as albuterol), is nearly universal for airway clearance. Conversely, novel modulator therapies restore function to select mutant CFTR proteins, offering a disease-modifying treatment. Recent trials of modulators targeting F508del-CFTR, the most common CFTR mutation, suggest that chronic $\beta$-agonist use may undermine clinical modulator benefits. We therefore sought to understand the impact of chronic or excess $\beta$-agonist exposure on CFTR activation in human airway epithelium. The present studies demonstrate a greater than $60 \%$ reduction in both wild-type and modulator-corrected F508del-CFTR activation following chronic exposure to short- and long-acting $\beta$-agonists. This reduction was due to reduced cellular generation of cAMP downstream of the $\beta-2$ adrenergic receptor- $G$ protein complex. Our results point towards a posttranscriptional reduction in adenylyl cyclase function as the mechanism of impaired CFTR activation produced by prolonged $\beta$-agonist exposure. $\beta$-Agonist-induced CFTR dysfunction was sufficient to abrogate VX809/VX770 modulation of F508del-CFTR in vitro. Understanding the clinical relevance of our observations is critical for CF patients using these drugs, and for investigators to inform future CFTR modulator drug trials.
\end{abstract}

Conflict of interest: The authors have declared that no conflict of interest exists.

Submitted: January 27, 2017

Accepted: January 23, 2018

Published: February 22, 2018

Reference information:

JCI Insight. 2018;3(4):e93029. https:// doi.org/10.1172/jici.insight.93029.

\section{Introduction}

Mutations in the gene encoding the cystic fibrosis transmembrane conductance regulator (CFTR) protein are responsible for the clinical symptoms and diagnosis of cystic fibrosis (CF) $(1,2)$. Over 2,000 diseasecausing mutations have been described to date, with F508del identified in the majority of CF patients (3). Most CF morbidity and mortality is due to pulmonary disease, wherein CFTR dysfunction leads to thickened mucus with airway surface liquid dehydration, airway obstruction, chronic infection, inflammation, and ultimately structural remodeling (2). Traditional pulmonary therapies in CF target the downstream effects of dysfunctional CFTR. With the advent of CFTR modulators that directly improve the function of mutant CFTR proteins, emerging therapies provide an opportunity to treat the cause of disease $(4,5)$. In patients homozygous for F508del-CFTR, lumacaftor (VX809) improves mutant CFTR trafficking to the cell surface, and when combined with ivacaftor (VX770), improves clinical outcomes including lung function $\left(\sim 3 \%\right.$ increase in forced expiratory volume in 1 second $\left.\left[\mathrm{FEV}_{1}\right]\right)$ and reduced risk of pulmonary exacerbation (6). While promising, these results are modest relative to ivacaftor monotherapy in patients with gating-class CFTR mutations $(7,8)$.

The CFTR is a member of the traffic ATPase protein family and is a chloride and bicarbonate channel. It is found in the apical plasma membrane of multiple epithelia, and is a critical regulator of ion and fluid balance $(1,9-11)$. The CFTR is primarily activated by PKA-dependent phosphorylation of its unique regulatory $(\mathrm{R})$ domain and ATP binding/hydrolysis at 2 nucleotide binding domains, which form a heterodimer to gate channel activity $(12,13)$. CFTR function, therefore, is dependent on local $3^{\prime}, 5^{\prime}$-cyclic adenosine monophosphate (cAMP) accumulation, which is highly regulated by spatially restricted proteins in the region of the CFTR (14). For example, CFTR activation via the $\beta$-2 adrenergic receptor ( $\beta 2 \mathrm{AR})$ is accomplished in part by C-terminal PDZ binding domains (present in both proteins) that plug the proteins into the scaffolding protein NHERF-1, effectively compartmentalizing the CFTR and the $\beta 2 A R$ in a regional signaling network (15). When bound by an appropriate agonist, $\beta 2 \mathrm{ARs}$ interact with $\mathrm{G}$ proteins 
(GPs), leading to dissociation of $\mathrm{G}_{\mathrm{S}} \alpha$ which activates adenylyl cyclase (AC) and catalyzes the conversion of ATP to cAMP $(16,17)$. Additional proteins such as A-kinase anchoring proteins help localize other critical proteins (e.g., AC, phosphodiesterases [PDEs], and multidrug resistance protein 4 [MRP4]) that titrate and compartmentalize cAMP levels and subsequent CFTR activity (18). While acute cAMP elevation is a necessary step in physiologic CFTR activation, loss of regulation leads to pathology such as cholera-induced diarrheal disease, where cholera toxin bypasses the cAMP regulatory network to chronically raise cAMP, drive CFTR activation, and produce life-threatening fluid losses $(19,20)$.

cAMP is produced by ACs, a group of enzymes with 9 identified transmembrane isoforms (21). Common features include 2 hydrophobic domains with 6 transmembrane spans and 2 cytoplasmic domains, where catalytic activity occurs. All 9 transmembrane AC isoforms are activated by the $\mathrm{G}_{\mathrm{S}} \alpha$ subunit of the GP, and all but AC9 are directly activated by the diterpene forskolin. This product of Coleus forskohli binds directly to the catalytic subunit of ACs, dimerizing the $C_{1}$ and $C_{2}$ domains and activating the enzyme independently of GP activity (22). Beyond this common mechanism, regulation of AC function is unique to each isoform, including modulation by inhibitory $\mathrm{G}_{\mathrm{i}} \alpha$, calcium, calcium-calmodulin complexes, and a variety of protein kinases (21).

It is well known that acute cAMP elevation activates CFTR in vitro and in vivo, but the impact of chronic cAMP elevation on CFTR regulation in airway cells has not been defined. This question is highly relevant, as $95 \%$ of $\mathrm{CF}$ patients are treated daily with $\beta 2 \mathrm{AR}$-agonists (which raise cAMP in airway epithelia) despite a recommendation of "insufficient evidence to support chronic use" from the CF Foundation $(23,24)$. Short-acting $\beta 2 \mathrm{AR}$-agonists, such as albuterol, are frequently used as a part of daily airway clearance regimens, and long-acting $\beta 2 \mathrm{AR}$-agonists (LABAs - such as formoterol) are also commonly prescribed (often in combination with inhaled corticosteroids to control asthma symptoms). This practice is based on limited clinical data, as well as in vitro human or in vivo animal model observations that single doses of $\beta 2 \mathrm{AR}$-agonists increase mucociliary clearance (MCC) (2527). Previous work on subacute and chronic $\beta 2 \mathrm{AR}$ activation has demonstrated myriad desensitization mechanisms, primarily including receptor uncoupling from the GP, receptor internalization, and PKA-induced downregulation of certain isoforms of AC (28-31). The downstream effects of these changes on CFTR function, however, are unclear. Notably, subgroup analysis of recent phase 3 trials of lumacaftor/ivacaftor in F508del-CFTR homozygous patients revealed an almost 3-fold higher improvement in $\mathrm{FEV}_{1}$ in the small subset of subjects who reported not receiving regular $\beta 2 \mathrm{AR}$-agonist therapy (6). In the current study, we examined relationships between chronic or excessive $\beta$-agonist exposure and subsequent CFTR activation in human airway epithelial cells (HAECs), including studies of wildtype CFTR (wtCFTR) and F508del-CFTR with and without correction by VX809. Our results indicate that chronic $\beta 2 \mathrm{AR}$ stimulation in HAECs downregulates cAMP generation by AC, limiting subsequent CFTR activation and reducing the corrective effects of VX809 on F508del-CFTR. These results identify a negative drug-drug interaction between $\beta 2 \mathrm{AR}$-agonists and lumacaftor/ivacaftor, with clinical implications that are potentially relevant to the majority of CF patients.

\section{Results}

Chronic $\beta 2 A R$-agonist exposure inhibits acute CFTR activation in HAECs. CFBE41o- cells stably transduced with wtCFTR and F508del-CFTR, in addition to primary HAEC monolayers, were studied as polarized monolayers on permeable supports as previously described (32). Notably, for all studies, forskolin and 3-isobutyl1-methylxanthine (IBMX) were used as a stimulus to generate CAMP and activate CFTR, unless otherwise noted. This method bypasses the effects of changes in the $\beta 2$ AR-GP complex and directly assesses AC activity and the downstream CFTR pathway. This stimulus was chosen in lieu of direct activators of the $\beta 2 \mathrm{AR}$ (e.g., isoproterenol) to avoid confounding related to the known mechanisms of $\beta 2 \mathrm{AR}$ downregulation after exposure to $\beta$-agonist drugs.

In wtCFTR ${ }^{+}$CFBE41o- cells and primary HAECs, 72-hour exposure to albuterol produced dosedependent reductions in the short-circuit current $\left(\mathrm{I}_{\mathrm{sc}}\right)$ in both model systems, with a greater than $70 \%$ reduction in CFTR-dependent conductance following chronic albuterol exposure at $10 \mu \mathrm{M}$ (Figure 1). This concentration approximates reported airway surface concentrations of albuterol achieved with standard dosing in vivo (33). Albuterol pretreatment did not change the baseline resistance of the cellular monolayers, or increase the change in $\mathrm{I}_{\mathrm{sc}}$ after establishing the basolateral-to-apical $\mathrm{Cl}^{-}$secretory gradient (i.e., low$\mathrm{Cl}^{-}$response), confirming that the majority of available wtCFTR at the beginning of our protocol was no 


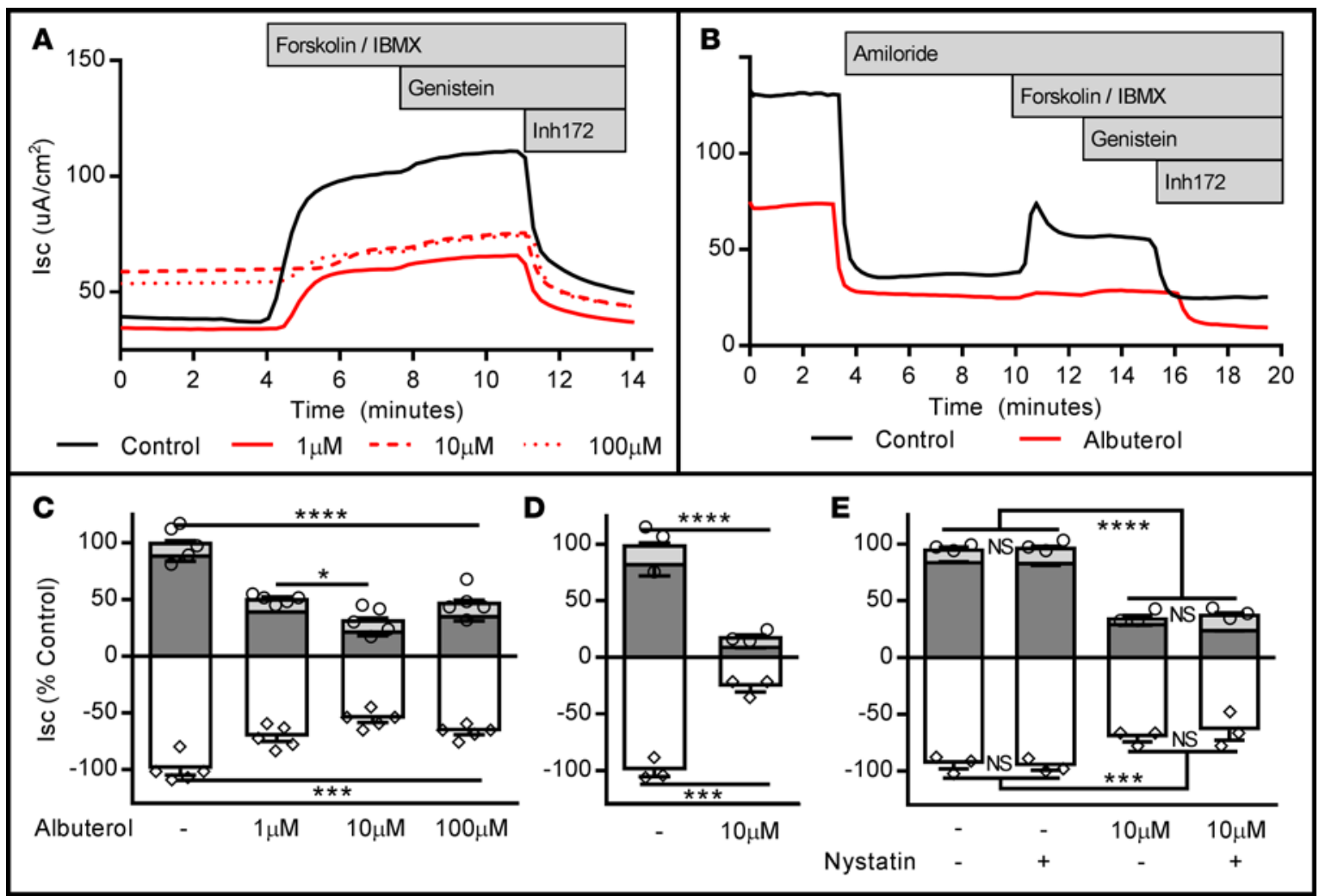

Figure 1. Seventy-two-hour albuterol exposure reduces stimulated apical CFTR activity in wtCFTR ${ }^{+}$CFBE410- cells and primary human airway epithelial cells (HAECs). wtCFTR ${ }^{+}$CFBE41o- cells and HAECs were exposed to albuterol in media for 72 hours, then mounted in Ussing chambers and CFTR function was assessed under voltage clamp conditions. (A) Representative short-circuit current $\left(\mathrm{I}_{\mathrm{sc}}\right)$ tracings from wtCFTR ${ }^{+}$CFBE410- cells chronically exposed to increasing doses of albuterol (amiloride perfusion is not shown for clarity as these cells do not demonstrate significant epithelial sodium channel expression; see ref. 62); aggregate data are presented in C ( $n=5$ inserts/condition; circles represent total CFTR activity [cAMP + genistein], diamonds represent

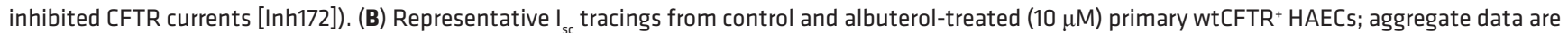
presented in $\mathbf{D}$ ( $n=3$ inserts/condition). (E) wtCFTR ${ }^{+}$CFBE410- cells treated with $10 \mu \mathrm{M}$ albuterol, studied with and without basolateral permeabilization (basolateral nystatin, $50 \mu \mathrm{g} / \mathrm{ml}$ ). Permeabilization does not modify inhibition of stimulated $\mathrm{I}_{\mathrm{sc}}$ with albuterol pretreatment ( $n=3$ inserts $/$ condition). All data were normalized to the control (no albuterol pretreatment) condition. Stimulation protocol was as follows: amiloride (100 $\mu \mathrm{M}$, not shown for CFBE410- cells), cAMP (10 $\mu \mathrm{M}$ forskolin/100 $\mu \mathrm{M}$ IBMX; dark gray bars), CFTR potentiator ( $50 \mu \mathrm{M}$ genistein; light gray bars), and CFTR inhibition (10 $\mu \mathrm{M}$ Inh172; white bars). All experiments are representative of studies repeated in duplicate or triplicate with similar results. Data presented represent the mean \pm SEM. ${ }^{*} P<0.05 ;{ }^{* *} P<0.005 ;{ }^{* * *} P<0.0005$; NS, nonsignificant by 2-way ANOVA with Tukey's multiple comparisons test.

longer active at the time of acute stimulation (Table 1). A small but significant increase in baseline current was seen in albuterol-pretreated wtCFTR ${ }^{+}$CFBE41o- cells (Table 1), although this was not recapitulated in F508del cells or in the primary wtCFTR cells. The nature of the increased baseline current produced by albuterol in the wtCFTR ${ }^{+}$CFBE41o- cells is unclear; importantly, subsequent CFTR activation and inhibition was consistently reduced in albuterol-treated cells (Figure 1).

Corollary studies in F508del-CFTR ${ }^{+}$CFBE41o- cells and primary HAECs (F508del/F508del) are presented in Figure 2. Similarly, chronic albuterol exposure at $10 \mu \mathrm{M}$ or higher resulted in a greater than $60 \%$ reduction in $\mathrm{I}_{\mathrm{sc}}$ compared with VX809-corrected levels. In primary HAECs, this treatment was sufficient to completely abrogate the corrective effect of VX809. In VX809-corrected F508del-CFTR ${ }^{+}$ CFBE41o- cells and HAECs, no difference was noted in baseline resistance, current, or in the change in $\mathrm{I}_{\mathrm{sc}}$ after establishing a basolateral-to-apical $\mathrm{Cl}^{-}$secretory gradient (i.e., low- $\mathrm{Cl}^{-}$response), confirming that the majority of available corrected F508del-CFTR at the beginning of our protocol was no longer active at the time of acute stimulation (Table 2).

To confirm that the inhibitory effect produced by albuterol pretreatment was localized to the apical cell membrane, wtCFTR ${ }^{+}$and VX809-corrected F508del-CFTR ${ }^{+}$CFBE41o- cells were studied following basolateral membrane permeabilization with nystatin $(50 \mu \mathrm{g} / \mathrm{ml})$ and stimulation with forskolin/IBMX $(10 \mu \mathrm{M} / 100 \mu \mathrm{M})$ (Figure 1E and Figure 2E). Similar albuterol-inhibitory effects were observed. 
Table 1. Seventy-two-hour exposure to albuterol does not impact baseline resistance or low-chloride change-inducible current in wtCFTR+ CFBE410- and primary HAECs

\begin{tabular}{|c|c|c|c|}
\hline & Untreated & Albuterol-treated & $\boldsymbol{P}$ \\
\hline \multicolumn{4}{|c|}{ Baseline Resistance $\left(\Omega \mathrm{cm}^{2}\right)$} \\
\hline CFBE410- & $199.6 \pm 50.3$ & $206.8 \pm 36.1$ & 0.91 \\
\hline HAEC & $396.6 \pm 32.2$ & $315.9 \pm 27.5$ & 0.09 \\
\hline \multicolumn{4}{|c|}{ Baseline $\mathrm{I}_{\mathrm{sc}}\left(\mu \mathrm{A} / \mathrm{cm}^{2}\right)$} \\
\hline CFBE410- & $-2.5 \pm 2.2$ & $4.3 \pm 0.8$ & 0.01 \\
\hline HAEC & $45.5 \pm 10.5$ & $23.8 \pm 5.0$ & 0.08 \\
\hline \multicolumn{4}{|c|}{ Low-Chloride $\mathrm{I}_{\mathrm{sc}}\left(\mu \mathrm{A} / \mathrm{cm}^{2}\right)$} \\
\hline HAEC & $22.2 \pm 6.7$ & $18.2 \pm 1.7$ & 0.54 \\
\hline
\end{tabular}

A statistically significant but small increase was noted in baseline short-circuit current $\left(I_{\text {sc }}\right)$ following albuterol pretreatment in CFBE410- cells, which is not recapitulated in primary cells, and did not impact the low-chloride change in $\mathrm{I}_{\mathrm{sc}}$ or subsequent CFTR activation/inhibition (per Figure 1). Data are from experiments depicted in Figure 1; $n=8$ CFBE410- inserts, 6 primary human airway epithelial cell (HAEC) inserts. All $P$ values by 2 -tailed $t$ test.

To determine the kinetics of albuterol exposure as a model of clinical $\beta 2 \mathrm{AR}$-agonist use, wtCFTR ${ }^{+}$and VX809-corrected F508del-CFTR ${ }^{+}$CFBE41o- cells were pretreated continuously or intermittently (1 hour exposure, twice daily) for 72 hours with $10 \mu \mathrm{M}$ albuterol or $1 \mu \mathrm{M}$ of the LABA formoterol. In wtCFTR ${ }^{+}$ cells, continuous albuterol exposure resulted in an equivalent reduction of forskolin/IBMX-stimulated $\mathrm{I}_{\mathrm{sc}}$ compared with intermittent or continuous formoterol exposure (Figure 3, A and B; it should be noted that in a replicate experiment, the intermittent albuterol group showed a significant reduction compared with control; however, in the experiment included, this reduction was a nonsignificant trend only; all other results were equivalent). In F508del-CFTR ${ }^{+}$cells, intermittent or continuous exposure to either LABA or short-acting $\beta$-agonist was sufficient to produce an equivalent reduction in forskolin/IBMX-stimulated CFTR-dependent $\mathrm{I}_{\mathrm{sc}}$ (Figure 3, C and D).

To examine the epithelial impact of chronic albuterol treatment $(10 \mu \mathrm{M})$ on downstream functions of the CFTR, we quantified mucociliary transport in wtCFTR ${ }^{+}$HAECs using time-lapse microscopy and single-particle tracking software. Qualitatively, movement of the airway surface mucus in control (untreated) cells was rapid and unidirectional, circling around the insert surface. In cells pretreated with either albuterol or the CFTR inhibitor GlyH101 $(50 \mu \mathrm{M})$, however, mucus movement was erratic and slow (Figure $4 \mathrm{~A}$ ). Pretreatment with albuterol or GlyH101 reduced MCC rates by approximately $60 \%$ despite equivalent ciliary motility (Figure 4B), suggesting that mucociliary transport was impaired independently of cilia activity. Data from F508del-CFTR ${ }^{+}$HAECs are not included, as baseline mucociliary transport in these cells (with or without VX809 treatment) was low and below the sensitivity of this assay.

Chronic $\beta 2 A R$-agonist exposure does not impact CFTR maturation or protein expression. To examine whether chronic $\beta 2 \mathrm{AR}$ stimulation impacted steady-state expression of the CFTR or CFTR maturation, wtCFTR ${ }^{+}$ (Figure 5A) and VX809-corrected F508del-CFTR ${ }^{+}$(Figure 5B) CFBE41o- cells were pretreated for 72 hours with $10 \mu \mathrm{M}$ albuterol, lysed, and immunoblotting for the CFTR was performed (normalized to $\beta$-actin). Chronic albuterol exposure $(10 \mu \mathrm{M})$ did not reduce normalized levels of mature CFTR in wtCFTR ${ }^{+}$(Figure 5C) or VX809-corrected F508del-CFTR ${ }^{+}$CFBE41o- cells (Figure 5D). Immunofluorescence staining for the $\beta 2 \mathrm{AR}$ and CFTR was then performed in both $\mathrm{wCFTR}^{+}$and VX809-corrected F508del-CFTR ${ }^{+}$primary HAECs in the presence or absence of albuterol exposure, and colocalization of CFTR and $\beta 2 \mathrm{AR}$ was quantified. As expected, in wtCFTR ${ }^{+}$cells, $\beta 2 \mathrm{AR}$ was present at the cell surface under control conditions (Figure 6A), but reduced following albuterol exposure (Figure 6B), with no apparent impact on CFTR localization. This resulted in a reduction of $\beta 2 \mathrm{AR}-\mathrm{CFTR}$ colocalization in analyses restricted to the apical surface of the cell (Figure 6C) or including the entire images (Figure 6D). The same pattern, though with reduced CFTR staining, was noted in VX809-corrected F508del-CFTR ${ }^{+}$homozygous primary HAECs (Figure 6, E-H).

Chronic $\beta 2 A R$-agonist exposure impairs $C A M P$ generation by $A C$. The results of studies summarized in Figures 5 and 6 indicate that cellular levels of mature CFTR were unlikely to explain the inhibitory effects of chronic albuterol on CFTR activation. We next examined whether chronic albuterol exposure inhibited cellular generation of cAMP following direct stimulation of AC. Similar to the functional CFTR data 


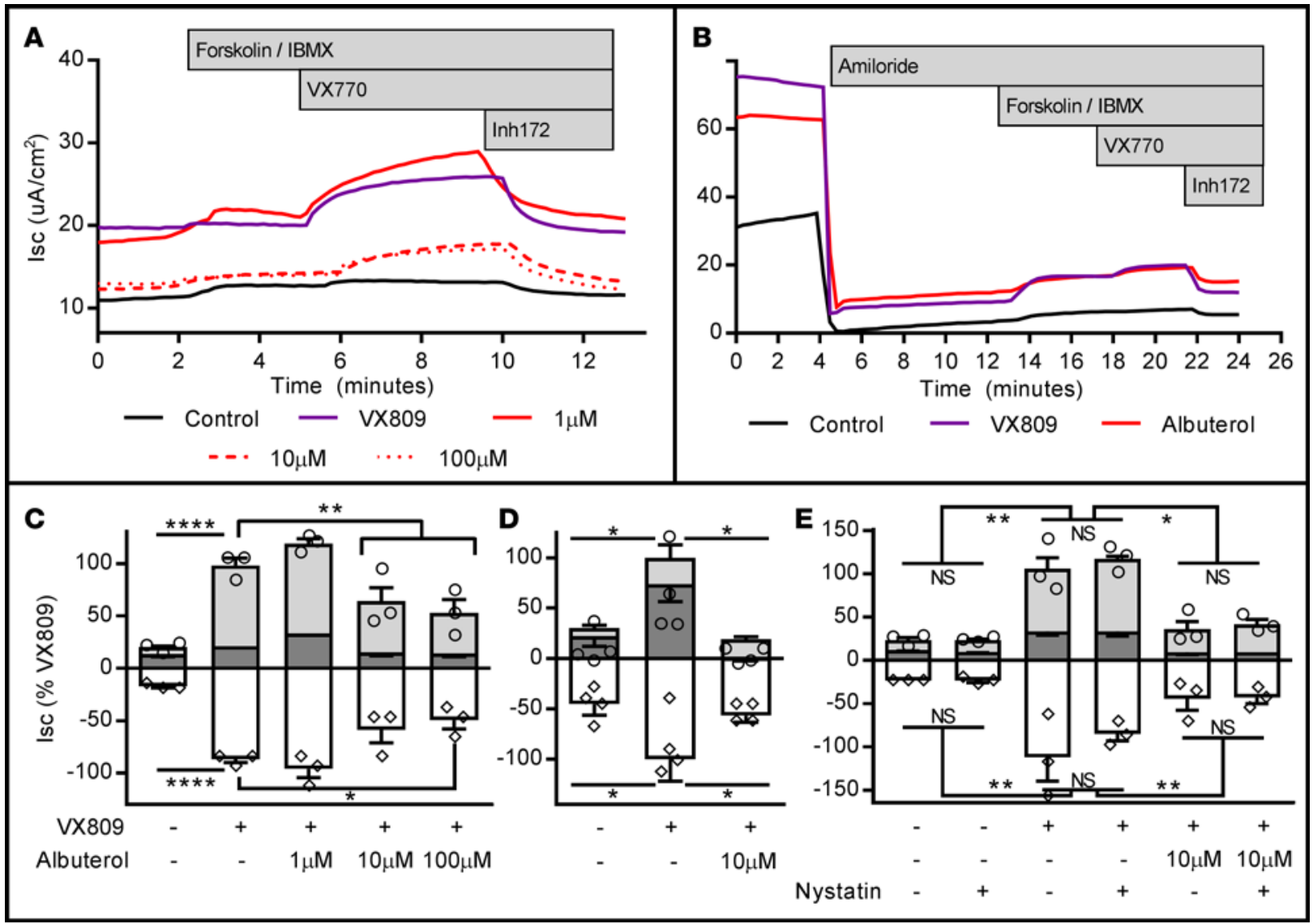

Figure 2. Seventy-two-hour albuterol exposure reduces stimulated apical CFTR activity in F508del-CFTR+ CFBE410- cells and primary human airway epithelial cells (HAECs) from F508del homozygous donors. F508del-CFTR+ CFBE410- cells and HAECs were exposed to VX809 and/or albuterol in media for 72 hours, then mounted in Ussing chambers and CFTR function was assessed under voltage clamp conditions. (A) Representative short-circuit current $\left(\mathrm{I}_{\mathrm{sc}}\right)$ tracings from VX809-corrected F508del-CFTR+ CFBE410- cells chronically exposed to increasing doses of albuterol; aggregate data are presented in C ( $n=3$ inserts/condition; circles represent total CFTR activity [cAMP + genistein], diamonds represent inhibited CFTR currents [Inh172]). (B) Representative $I_{\mathrm{Sc}}$ tracings from control, VX809-, and VX809 + albuterol-treated $(10 \mu \mathrm{M})$ primary F508del-CFTR ${ }^{+}$HAECs; aggregate data are presented in D, where pretreatment with albuterol completely abrogated the positive effects of VX809 treatment ( $n=4$ inserts/condition). (E) F508del-CFTR+ CFBE410- cells corrected with VX809 and treated with $10 \mu \mathrm{M}$ albuterol, studied with and without basolateral permeabilization (basolateral nystatin, $50 \mu \mathrm{g} / \mathrm{ml}$ ). Permeabilization does not modify inhibition of stimulated $\mathrm{I}_{\mathrm{sc}}$ with albuterol pretreatment ( $n=3$ inserts/condition). All data were normalized to the VX809 (no albuterol pretreatment) condition. Stimulation protocol was as follows: amiloride (100 $\mu \mathrm{M}$, not shown for CFBE410- cells), cAMP (10 $\mu \mathrm{M}$ forskolin/100 $\mu \mathrm{M}$ IBMX; dark gray bars), CFTR potentiator ( $1 \mu \mathrm{M}$ VX770; light gray bars), and CFTR inhibition ( $10 \mu \mathrm{M}$ Inh172; white bars). All experiments are representative of studies repeated in duplicate or triplicate with similar results. Data presented represent the mean $\pm \mathrm{SEM} .{ }^{*} P<0.05 ;{ }^{* *} P<0.01 ;{ }^{* * * *} P<0.0005 ;$ NS, nonsignificant by 2-way ANOVA with Tukey's multiple comparisons test.

presented in Figures 1 and 2, chronic albuterol exposure in CFBE41o- cells reduced acute cAMP generation following stimulation by approximately $60 \%$ (wtCFTR $^{+}$, Figure $7 \mathrm{~A}$ ) to approximately $80 \%$ (F508del$\mathrm{CFTR}^{+}$, Figure 7B) compared with the untreated, forskolin/IBMX-stimulated conditions.

We next performed a series of studies testing whether chronic $\beta 2 A R$ stimulation had measurable functional effects on other proteins that contribute to spatial cAMP levels and local CFTR regulation. Our first studies examined phosphodiesterase 4 (PDE-4), as PDE-4 isotypes have been demonstrated to acutely regulate CFTR activity $(34,35)$. To assess the functional contribution of PDE-4 to the albuterol-induced CFTR dysfunction demonstrated above, rolipram (PDE-4-specific inhibitor, $10 \mu \mathrm{M}$ ) was added to cells acutely in a Ussing chamber. (Note that the experiments described in all figures above also utilize IBMX, a nonspecific PDE inhibitor; rolipram studies were completed to ensure maximal inhibition of PDE-4 specifically.) CFTRdependent $\mathrm{I}_{\mathrm{sc}}$ was not restored with acute rolipram treatment in either wtCFTR ${ }^{+}$(Supplemental Figure 1A; supplemental material available online with this article; https://doi.org/10.1172/jci.insight.93029DS1) or VX809-corrected F508del-CFTR ${ }^{+}$cells (Supplemental Figure 1B) pretreated with albuterol (10 $\mu \mathrm{M}, 72$ hours). These results support the hypothesis that PDE-4 upregulation is not responsible for the reduction of cAMP generation and CFTR activation produced by pretreatment with albuterol. In similar experiments examining 
Table 2. Seventy-two-hour exposure to albuterol does not impact baseline current, resistance, or low-chloride change-inducible current in F508del-CFTR+ CFBE410- and primary HAECs

\begin{tabular}{|c|c|c|c|}
\hline & VX809-treated & VX809 and Albuterol-treated & $\boldsymbol{P}$ \\
\hline \multicolumn{4}{|c|}{ Baseline Resistance $\left(\Omega \mathrm{cm}^{2}\right)$} \\
\hline CFBE410- & $433.9 \pm 49.5$ & $534.2 \pm 72.5$ & 0.27 \\
\hline HAEC & $493.4 \pm 149.5$ & $502.2 \pm 188.4$ & 0.97 \\
\hline \multicolumn{4}{|c|}{ Baseline $\mathrm{I}_{\mathrm{sc}}\left(\mu \mathrm{A} / \mathrm{cm}^{2}\right)$} \\
\hline CFBE410- & $2.0 \pm 1.5$ & $4.5 \pm 1.4$ & 0.25 \\
\hline HAEC & $31.7 \pm 7.7$ & $19.9 \pm 7.6$ & 0.30 \\
\hline HAEC & $24.9 \pm 11.9$ & $26.3 \pm 8.3$ & 0.93 \\
\hline
\end{tabular}

Data are from experiments depicted in Figure 2; $n=8$ CFBE410- inserts, 8 primary human airway epithelial cell (HAEC) inserts. All $P$ values by 2-tailed $t$ test. $I_{\mathrm{sc}}$, short-circuit current.

if increased MRP4 function contributed to reduced cAMP generation, albuterol-pretreated cells were exposed to the MRP4 inhibitor MK571 $(20 \mu \mathrm{M})$ prior to routine CFTR-dependent $\mathrm{I}_{\mathrm{sc}}$ testing. Acute MRP4 blockade did not rescue CFTR activation by forskolin/IBMX in either wtCFTR ${ }^{+}$(Supplemental Figure 1C) or VX809corrected F508del-CFTR ${ }^{+}$(Supplemental Figure 1D) cells pretreated with albuterol. In wtCFTR ${ }^{+}$cells, MRP4 blockade further decreased CFTR activation; this phenomenon has been previously noted in airway epithelial cells, and may relate to $\mathrm{PGE}_{2}$ efflux through MRP4 (36). Regardless, our results indicate that MRP4 inhibition was not sufficient to restore CFTR activation following chronic $\beta 2 \mathrm{AR}$ stimulation.

To confirm that reduced cAMP generation by AC was responsible for the observed defect in CFTR activation, we set up experiments to determine if direct and controlled cAMP exposure could rescue CFTR activity following albuterol pretreatment. In these studies, cells were stimulated with cell-permeant 8-bromoadenosine $3^{\prime}, 5^{\prime}$-cyclic monophosphate $(100 \mu \mathrm{M})$ to activate the CFTR in lieu of forskolin/ IBMX, testing if the observed defect in CFTR activity could be overcome with exogenous cAMP. In both wtCFTR $^{+}$(Figure 8, A and B) and VX809-corrected F508del-CFTR ${ }^{+}$cells (Figure 8, D and E), stimulation with cell-permeant cAMP rescued the albuterol-induced CFTR dysfunction, implicating defects in cAMP generation as the cause of the observed effects. Dose-response analysis of albuterol-treated cells revealed a small, but significant increase in CFTR function following albuterol pretreatment with low-level cAMP stimulation in wtCFTR ${ }^{+}$cells only (Figure 8C), with no difference at higher doses in wtCFTR ${ }^{+}$ cells or at any dose in VX809-corrected F508del-CFTR ${ }^{+}$cells (Figure 8F).

Chronic $\beta 2 A R$-agonist exposure does not modify $A C$ isoform expression. We next evaluated the effect of chronic albuterol exposure on gene expression in primary HAECs, including wtCFTR ${ }^{+}$and VX809-corrected HAECs exposed to vehicle or $10 \mu \mathrm{M}$ albuterol for 72 hours. RNA sequencing (RNAseq) was performed and differential gene expression was quantified within cells (comparing albuterol to control conditions in wtCFTR ${ }^{+}$cells and albuterol/VX809 to VX809 alone in F508del/F508del-CFTR ${ }^{+}$monolayers). Minimal transcriptional change occurred in either line, with less than 10 genes up- or downregulated by more than 2-fold; no gene expression was changed by more than 2-fold in both wtCFTR ${ }^{+}$and VX809-corrected F508del-CFTR ${ }^{+}$HAECs (Supplemental Figure 2). Expression levels of all members of the CFTR activation pathway, including all identified AC isoforms, did not demonstrate significant changes in expression following albuterol exposure.

Transcripts from all AC isoforms, with the exception of AC5, were identified in both cell lines. The most highly expressed isoforms included AC3, AC4, AC6, AC7, and AC9 (Supplemental Figure 3). These expression patterns were similar between wtCFTR ${ }^{+}$and VX809-corrected F508del-CFTR ${ }^{+}$cells (with the exception of $\mathrm{AC} 3$, which was increased in $\mathrm{F}^{2} 08 \mathrm{del}^{+}$cells), and none were changed with albuterol exposure.

\section{Discussion}

Cell signaling through surface receptors to activate AC and generate cAMP is a critical step in numerous physiologic processes $(16,17,21)$, including activation of the CFTR. CFTR function is intrinsically tied to $\beta 2 A R s$ and cAMP signaling through protein-protein interactions, creating a local regulatory network to carefully control local cAMP levels and spatial activation of CFTR $(15,18,37)$ 


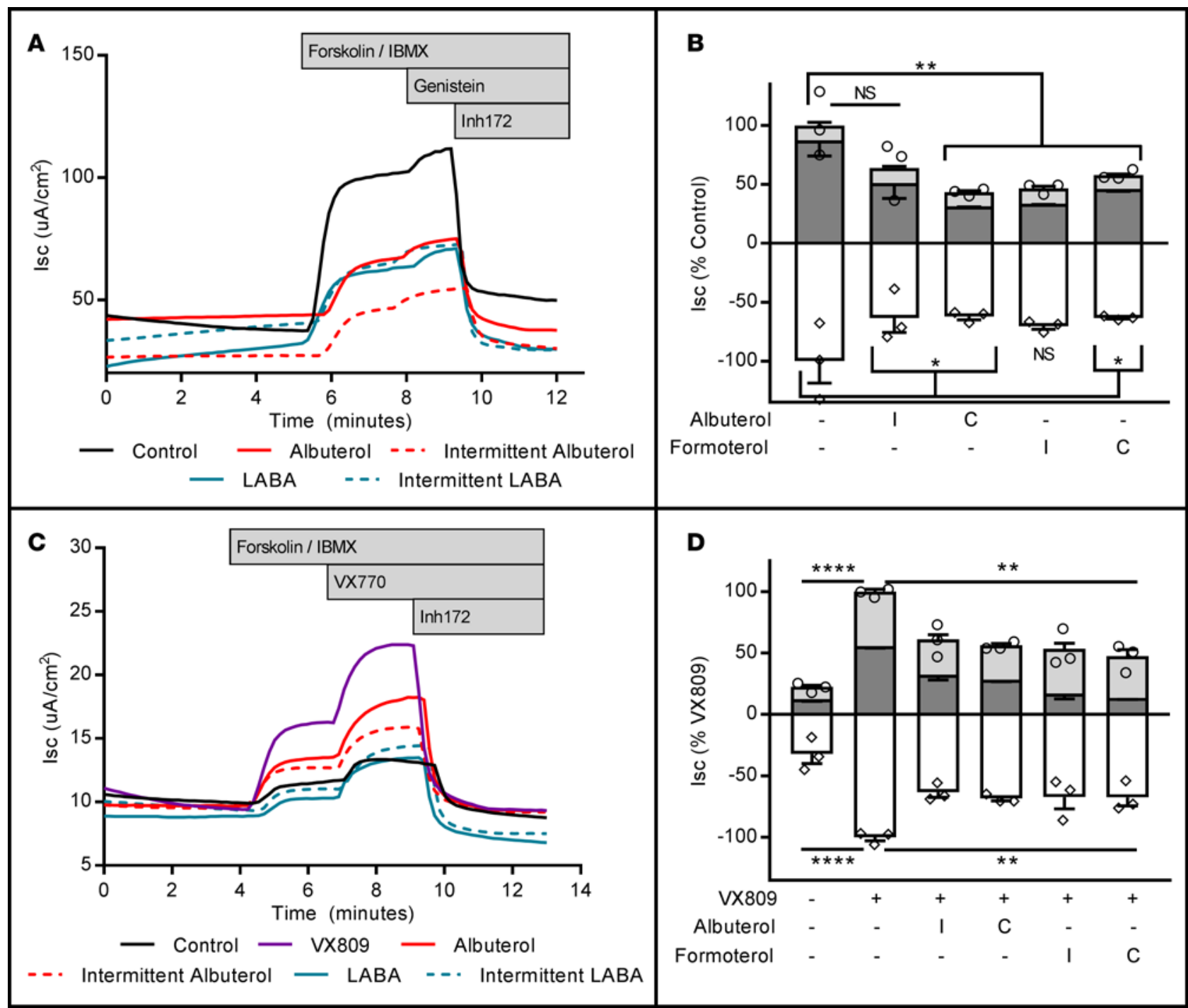

Figure 3. Continuous exposure to albuterol reduces stimulated CFTR activity in a fashion similar to continuous or intermittent dosing with formoterol. wtCFTR ${ }^{+}$and VX809-corrected F508del-CFTR+ CFBE410- cells were exposed to either continuous or intermittent (1 hour, twice daily) albuterol (10 $\left.\mu \mathrm{M}\right)$ or the long-acting $\beta 2 A R$-agonist formoterol (LABA; $1 \mu \mathrm{M}$ ) for 72 hours. Cells were mounted in Ussing chambers and CFTR function was assessed under voltage clamp conditions. (A) Representative short-circuit current $\left(I_{\mathrm{ss}}\right)$ tracings in wtCFTR+ CFBE410- cells exposed for 72 hours to either continuous (C) or intermittent (I) albuterol or formoterol; aggregate data are presented in B ( $n=4$ inserts/condition; circles represent total CFTR activity [CAMP + genistein], diamonds represent inhibited CFTR currents [Inh172]). (C) Representative $\mathrm{I}_{\mathrm{sC}}$ tracings in VX809-treated F508del-CFTR ${ }^{+}$CFBE410- cells exposed for 72 hours to either continuous (C) or intermittent (I) albuterol or formoterol; aggregate data are presented in $\mathbf{D}$ ( $n=4$ inserts/condition). All data were normalized to the control or VX809 (no albuterol pretreatment) condition. Stimulation protocol was as follows: amiloride (100 $\mu \mathrm{M}$, not shown), cAMP (10 $\mu \mathrm{M}$ forskolin/100 $\mu \mathrm{M}$ IBMX; dark gray bars), CFTR potentiator (50 $\mu \mathrm{M}$ genistein for wtCFTR ${ }^{+}, 1 \mu \mathrm{M}$ VX770 for F508del-CFTR ${ }^{+}$cells; light gray bars), and CFTR inhibition (10 $\mu \mathrm{M}$ Inh172; white bars). All experiments are representative of studies repeated in duplicate or triplicate with similar results. Data presented represent the mean \pm SEM. ${ }^{*} P<0.05$; ${ }^{* *} P<0.01$; ${ }^{* * *} P<0.0005$; NS, nonsignificant by 2 -way ANOVA with Tukey's multiple comparisons test.

Our results in heterologous CFTR-expressing human airway cells and primary HAECs indicate that chronic exposure to the $\beta$-agonists albuterol and formoterol is sufficient to reduce subsequent cAMP generation following direct AC stimulation (forskolin) and nonspecific PDE inhibition (IBMX). This results in reduced activation of wtCFTR and VX809-corrected F508del-CFTR, and reduced MCC in $\mathrm{wtCFTR}^{+}$primary airway epithelia. Studies to determine the mechanism of our observations point towards a negative relationship between chronic $\beta 2 \mathrm{AR}$ stimulation and $\mathrm{AC}$ activity, which can be overcome when AC stimulation is bypassed directly with cell-permeant cAMP. These observations are of direct clinical relevance to the care of CF patients treated with CFTR modulators and raise a number of critical considerations. 


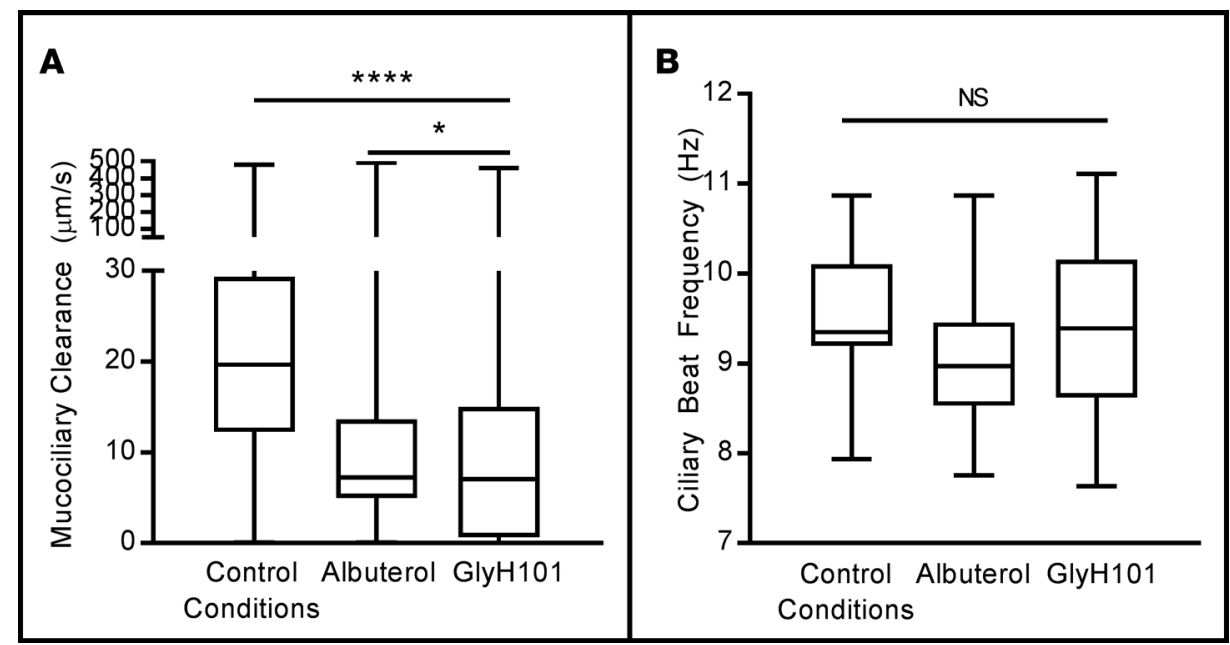

Figure 4. Chronic albuterol exposure reduces mucociliary clearance (MCC) in wtCFTR+ primary human airway epithelial cells (HAECs). wtCFTR ${ }^{+}$primary HAECs were exposed to either albuterol $(10 \mu \mathrm{M})$ or the CFTR inhibitor GlyH101 $(50 \mu \mathrm{M})$ for 72 hours. Fluorescent microbeads were placed on the apical surface of the cells 72 hours prior to testing, allowing airway surface liquid to return to equilibrium. MCC rate was then calculated as speed of microbead transport, measured with fluorescence microscopy and single-particle tracking, and ciliary beat frequency (CBF) was measured with high-speed videomicroscopy. Seventy-two-hour pretreatment with either albuterol (10 $\mu \mathrm{M})$ or the CFTR inhibitor $\mathrm{GlyH} 101(50 \mu \mathrm{M})$ resulted in a greater than $60 \%$ reduction in MCC rate (A, $n=3$ inserts, $>3,000$ measurements per insert). Despite reduced mucus movement, CBF was not affected (B, $n=3$ inserts, 20 measurements per insert). These experiments are representative of studies repeated in triplicate, with similar results. Data presented represent the mean $\pm S E M$. Box whiskers range from minimum to maximum, with no data excluded. Boundaries of boxes represent 25th (lower) and 75th (upper) percentiles, while the central bar represents the median. ${ }^{*} P<0.05$; ${ }^{* * *} P<0.0005$; NS, nonsignificant by 1-way ANOVA with Tukey's multiple comparisons test.

Albuterol and LABA use is nearly ubiquitous in CF, with $96 \%$ of patients using at least one of these therapies chronically (24). The acute benefits of $\beta 2 A R$-agonists on airway clearance are readily evident, with known improvements in bronchodilation and mucus clearance. There are limited data, however, regarding the chronic use of these drugs in $\mathrm{CF}$, with small and contradictory randomized controlled trials to date $(26,27)$. Moreover, these studies predate CFTR modulator therapies, and interactions between chronic $\beta 2 \mathrm{AR}$-agonist stimulation and CFTR modulator function have not been reported. Prior studies in F508del homozygous HAECs show an improvement in F508del-CFTR function to approximately $14 \%$ of wtCFTR after treatment with VX809, which is essentially doubled by the addition of VX770 (38). This level of functional rescue is associated with clinical improvement in lung function and risk of pulmonary exacerbations (6). Critically, in the present studies, we observed that corrected F508del-CFTR activation was negatively impacted by chronic $\beta 2 A R$-agonist exposure. We speculate that the observed reduction in CFTR function following chronic $\beta 2 A R$ activation would likely have little impact in healthy individuals with normal baseline CFTR function and ion transport. Similarly, patients with minimal or no baseline CFTR function (e.g., F508del homozygotes) would not likely be impacted by treatments that reduce CFTR activation. In CF patients on modulator therapies, however, our data suggest a mechanism by which excessive use of $\beta 2 A R$-agonists may undermine the beneficial effects of pharmacologic F508del correction. Indeed, subgroup analysis of the phase 3 trials of lumacaftor/ivacaftor suggested a 3 -fold increase in $\mathrm{FEV}_{1}$ benefit for the small subset of subjects naive to $\beta 2 \mathrm{AR}$-agonist therapy; similar results were noted in phase 3 trials of tezacaftor/ivacaftor, confirming that this effect will remain a concern for future drugs as well $(6,39,40)$. It is noteworthy that there was great variability in this observation, with wide confidence intervals surrounding the mean effect. Moreover, numerous CFTR modulators are currently in clinical trials, a setting wherein this effect could be a significant confounder. In a recent example, a phase 3 trial of the read-through agent ataluren for those harboring premature termination codons in the CFTR failed to produce improvement in $\mathrm{FEV}_{1}$. Subgroup analysis suggested negative interactions between ataluren and inhaled tobramycin, a highly utilized, chronic, traditional therapy in CF (41). In another recent study, Nichols and colleagues reported that negative interactions between inhaled tobramycin and oral azithromycin may have impacted the results of a study 


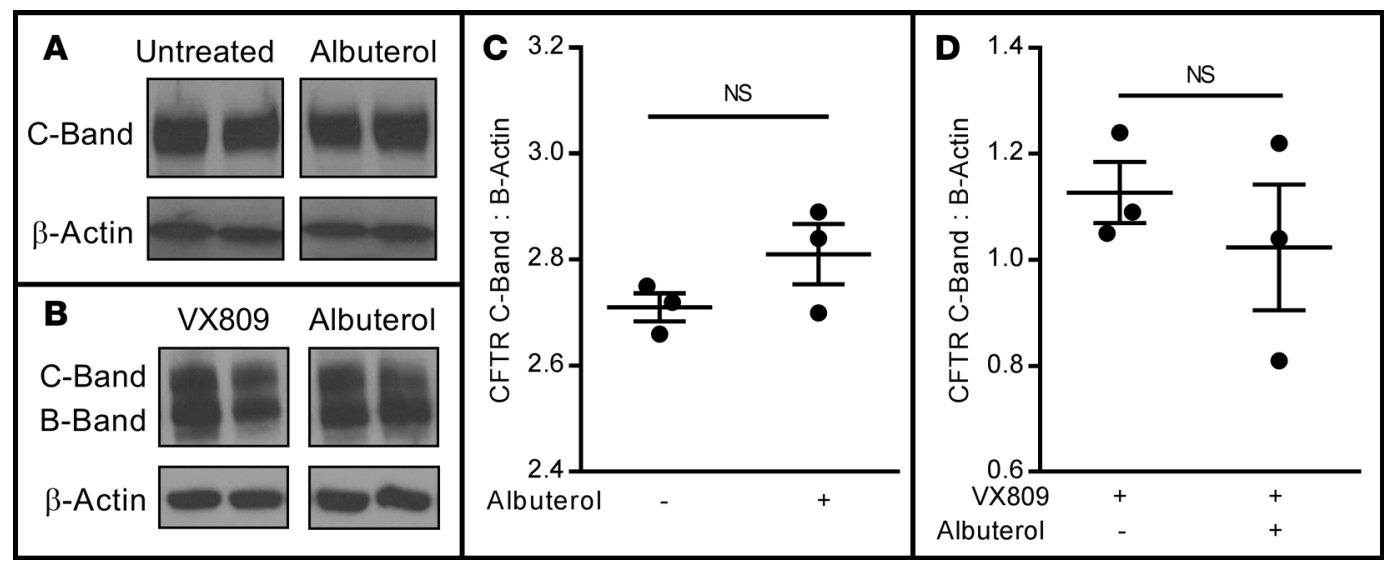

Figure 5. Chronic albuterol exposure does not reduce CFTR expression or maturation in wtCFTR ${ }^{+}$or F508del-CFTR ${ }^{+}$CFBE410- cells. wtCFTR $^{+}$and VX809-corrected F508del-CFTR+ CFBE410- cells were continuously exposed to albuterol (10 $\mu \mathrm{M})$, lysed, and CFTR protein was isolated by Western blot. Both C- and B-band forms were quantified by densitometry (relative to $\beta$-actin). Representative CFTR Western blots of wtCFTR ${ }^{+}$(A) and VX809-corrected F508del-CFTR ${ }^{+}$(B) CFBE410- cells following selective 72-hour pretreatment with albuterol. Each lane represents a separate insert; within each donor, all lanes were run in the same gel, but were noncontiguous. Densitometry corrected to $\beta$-actin shows no difference in mature C-band CFTR levels in either wtCFTR $^{+}$(C) or F508del-CFTR ${ }^{+}$(D) CFBE410- cells ( $n=3$ inserts/lanes). These experiments are representative of studies repeated in duplicate with similar results. Data presented represent the mean \pm SEM. NS, nonsignificant by 2-tailed $t$ test.

comparing nebulized antibiotics to treat CF patients chronically infected with Pseudomonas aeruginosa (42). Given the polypharmacy of CF care, identification and mechanistic understanding of such interactions must be better defined to guide clinical trial designs in $\mathrm{CF}$ patients.

We have shown that $\beta 2 \mathrm{AR}$-induced CFTR dysfunction relates to downstream attenuation of the $\beta 2 \mathrm{AR} / \mathrm{GP} / \mathrm{AC} / \mathrm{CFTR}$ axis, specifically at the site of AC catalytic activity. Homologous desensitization of this axis through changes in the $\beta 2 \mathrm{AR}$ is a well-described phenomenon $(30,31,43)$. These changes, however, are not implicated in our results, as all studies herein utilized a downstream agonist - forskolin - to stimulate AC activity, bypassing changes in the proximal $\beta 2 A R-G P$ unit. Desensitization of the $\beta 2 A R /$ GP/AC system in airway epithelial cells has been demonstrated following chronic activation with forskolin or PGE2, though this study used a proximal stimulus (isoproterenol), which may reflect only changes in the $\beta 2 \mathrm{AR}$ and not the effector (AC) (a key difference from the present work; see ref. 44).

The demonstrated reduction in acute cAMP generation observed following chronic albuterol exposure generally correlated with reduced CFTR function, and application of exogenous cAMP (bypassing AC activity) rescued CFTR activation. Interestingly, there was a small benefit of chronic albuterol exposure in $\mathrm{wtCFTR}^{+}$cells at very low doses of exogenous cAMP (Figure 8C), which may reflect prior studies demonstrating an increase in cell surface CFTR following activation of the $\beta 2 \mathrm{AR}$ (37). This benefit was lost as doses of exogenous cAMP increased, and importantly was not demonstrated in VX809-corrected F508del cells. Taken together, these results strongly suggest reduced generation of cellular cAMP as the proximate cause of the CFTR chloride transport defect. Nonetheless, the stoichiometry of cell signaling is highly variable, and assumptions of a 1:1 interaction should be taken cautiously (45). Moreover, cAMP signaling is tightly regulated in spatial complexes; as such, measured whole-cell levels may not directly reflect this critical driver of CFTR function, and exogenous CAMP application likely bypasses these regulatory mechanisms (18). In addition to AC, there are a number of critical mediators of cAMP levels that we examined to explain our findings. PDEs are responsible for the breakdown of cAMP; in the airway epithelium, PDE4 is the predominant isoform, allowing for precise control of cAMP levels at the local, perimembranous environment (34). Similarly, MRP4 has been demonstrated to function as a cAMP efflux pump in intestinal cells, regulating spatial cAMP concentration at the cell surface $(46,47)$. Functional inhibition of both PDE4 and MRP4, however, did not reverse albuterol-induced CFTR dysfunction (Supplemental Figure 1), making it unlikely that either of these regulators of spatial cAMP levels are implicit in our results.

The AC isoform(s) directly responsible for CFTR activation in the human airway epithelium are currently unknown. Our RNAseq data identify several candidate isoforms (Supplemental Figure 3), and show that there were no measurable effects of $\beta 2 \mathrm{AR}$ exposure on any $\mathrm{AC}$ isoform expression, pointing instead towards posttranscriptional modification. Notably, chronic PKA stimulation has been reported to downregulate AC5 


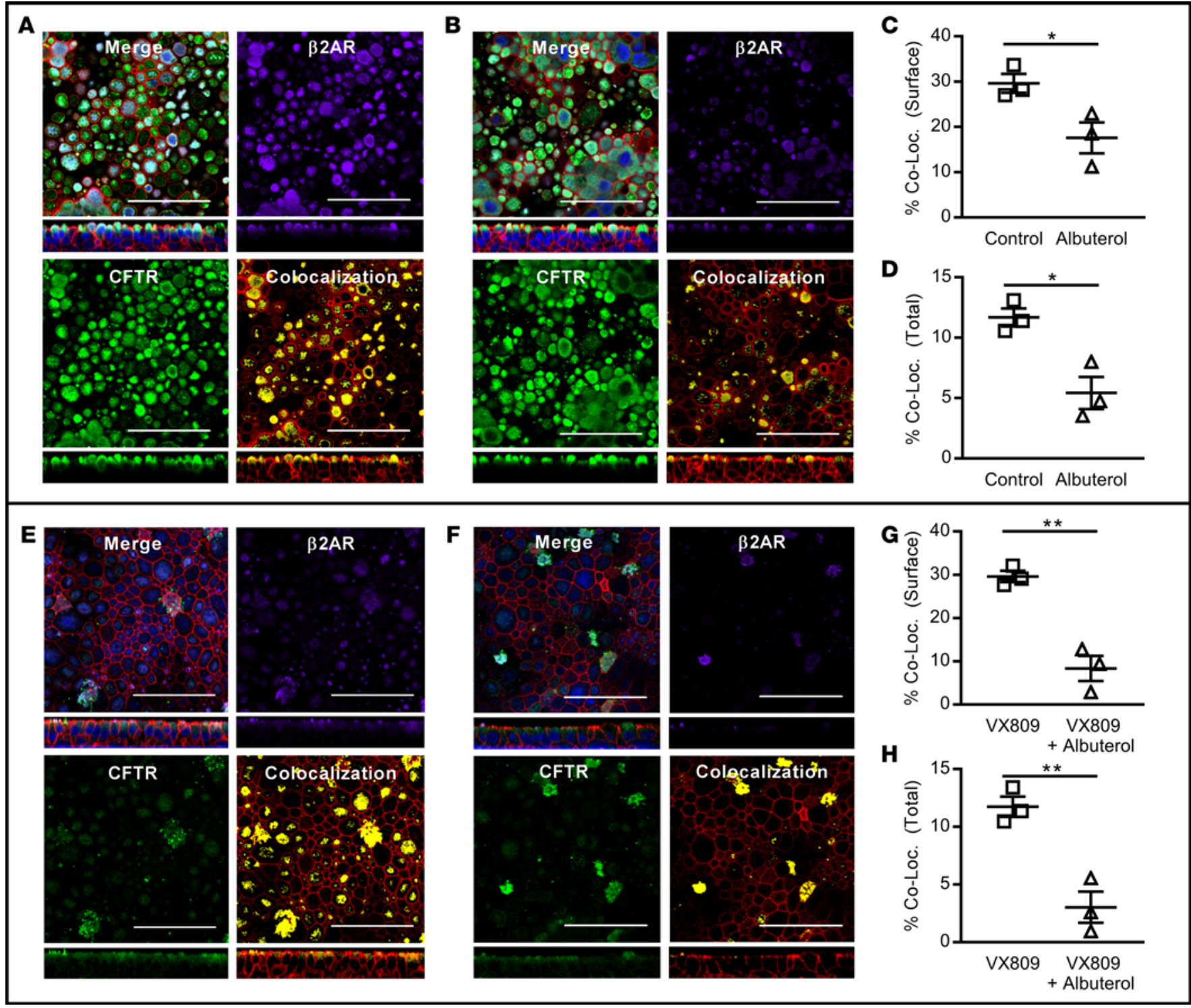

Figure 6. Chronic albuterol exposure reduces cell surface $\beta 2 A R$, but not CFTR, localization in wtCFTR ${ }^{+}$and VX809-corrected F508del-CFTR ${ }^{+}$primary human airway epithelial cells (HAECs). wtCFTR ${ }^{+}$and VX809-corrected F508del-CFTR ${ }^{+}$HAECs were exposed to albuterol (10 $\left.\mu \mathrm{M}\right)$ for 72 hours. Cells were then fixed and immunofluorescence for CFTR (green), F-actin (red), and $\beta-2$ adrenergic receptor ( $\beta 2 A R$ ) (purple) was performed. Colocalization analyses were performed to determine the colocalization of CFTR and $\beta 2 A R$ at the cell surface (manually delineated) and the entire captured images. Colocalization analysis included both single images in the $x-y$ and $x$-z planes (shown) and 3 -dimensional reconstruction images in the $x-y-z$ plane. wtCFTR ${ }^{+}$HAECs are shown under control conditions (A) and with albuterol pretreatment (B), demonstrating reduced apical $\beta 2 A R$ staining with albuterol pretreatment. Colocalization analysis of a manually delineated apical surface segment or the native images (C and $\mathbf{D}$, respectively; $n=3$ ), confirms reduced colocalization of wtCFTR and $\beta 2 A R$ following albuterol exposure. Similarly, VX809-corrected F508del-CFTR ${ }^{+}$HAECs are shown in the absence (E) or presence of albuterol (F). Equivalent colocalization analysis again demonstrates reduced CFTR $/ \beta 2 A R$ colocalization in both the apical surface $(\mathbf{C})$ and the entire images $(\mathbf{H}) ; n=3$ for both analyses. Data presented represent the mean \pm SEM. Scale bars: $25 \mu \mathrm{m}$. ${ }^{*} P<0.05$; ${ }^{*} P<0.01$ by 2 -tailed $t$ test.

and AC6 $(28,29)$. It is possible that our model of chronic albuterol exposure could result in excessive PKA activation through accumulation of $32 \mathrm{AR}$-stimulated cAMP, and may ultimately downregulate AC6 activity. It is important to note, however, that this phenomenon would be highly attenuated by internalization of the $\beta 2 \mathrm{AR}$ and well-described desensitization of that cAMP generation pathway, making this mechanism less likely. Preliminary work from our lab to clarify the role of AC6 in this phenomenon piloted siRNA knockdown of AC6 expression in both primary HAECs and CFBE41o- cells, but conclusions have been limited due to inconsistent effects on AC6 protein levels. To further define the specific AC isoforms responsible for CFTR activation and in the observed $\beta 2 \mathrm{AR}$-induced CFTR dysfunction, additional studies will be required, which are beyond the scope of the current work. Preliminary work investigating the effects of chronic stimulation 


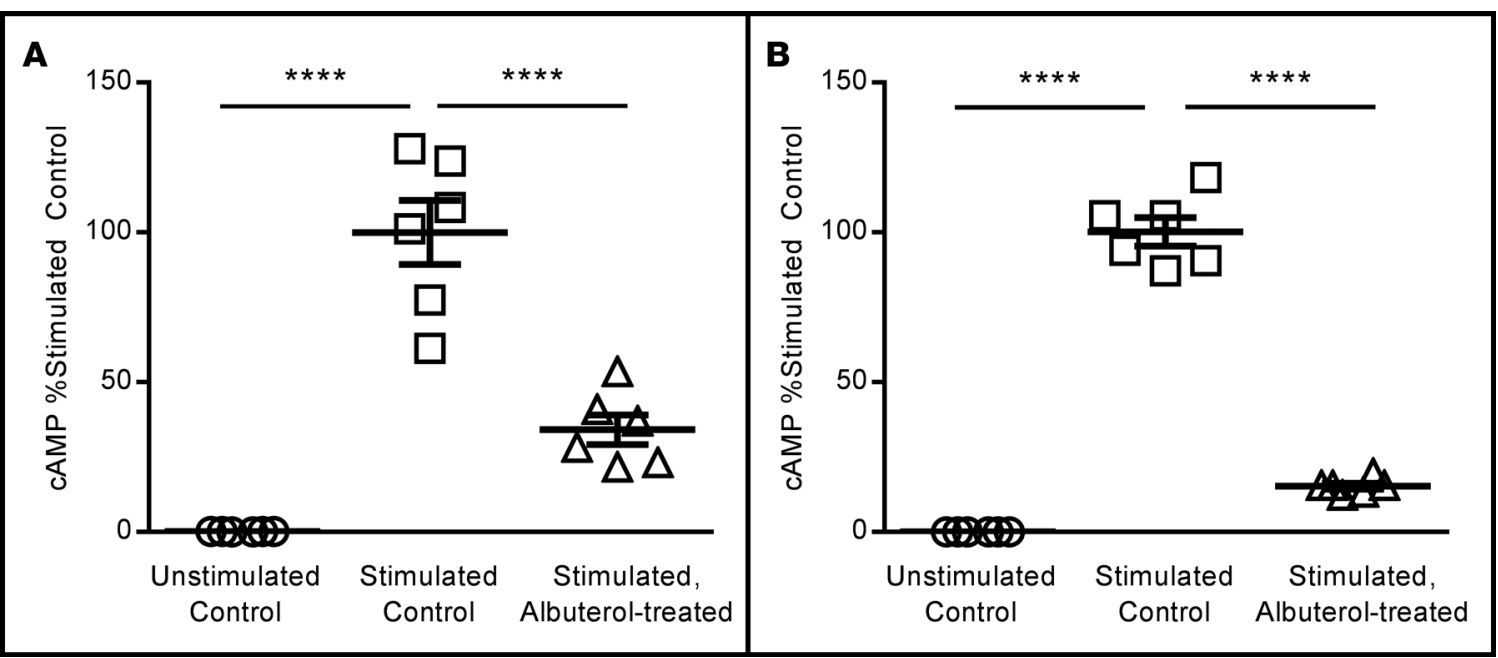

Figure 7. Chronic albuterol exposure reduces forskolin/IBMX-stimulated generation of CAMP in wtCFTR ${ }^{+}$and F508del-CFTR ${ }^{+}$CFBE410- cells. wtCFTR ${ }^{+}$ and F508del-CFTR ${ }^{+}$CFBE41o- cells were pretreated with albuterol $(10 \mu \mathrm{M})$ for 72 hours, then stimulated to produce cAMP with $10 \mu \mathrm{M}$ forskolin/100 $\mu \mathrm{M}$ IBMX (10 minutes). cAMP levels were then analyzed by colorimetric ELISA. wtCFTR ${ }^{+}(\mathbf{A}, n=6)$ and F508del-CFTR ${ }^{+}(\mathbf{B}, n=6)$ CFBE410- cells produce minimal CAMP in the absence of either pretreatment or stimulation (open circles). Stimulation with $10 \mu \mathrm{M}$ forskolin/100 $\mu \mathrm{M}$ IBMX in untreated cells leads to cAMP production (open squares), which is reduced by more than $60 \%$ in cells pretreated for 72 hours with albuterol (open triangles). All studies are internally normalized to control conditions to allow for comparisons. Both experiments are representative of studies repeated in duplicate with similar results. Data presented represent the mean \pm SEM. $n=6$ wells per condition. ${ }^{* * *} P<0.0005$ by 1 -way ANOVA with Tukey's multiple comparisons test.

with other cAMP-generating agonists (e.g., adenosine, forskolin) have demonstrated similar impairments in CFTR function, suggesting a common target in the CFTR activation pathway regardless of stimulus (48). There are also additional potential contributors to consider beyond cAMP signaling alone, such as direct interactions between subunits of the GP and CFTR or alterations in cell-surface CFTR levels, though this is less likely given the negative effects of albuterol on CFTR activation were rescued by stimulation with exogenous cAMP (Figure 8) (49-54).

There are well-described spatial mechanisms of CFTR regulation, including colocalization of $\beta 2 \mathrm{AR}$ GP-AC, CFTR, and PKA by NHERF1 $(18,47)$. Disruption of this spatial relationship via chronic $\beta 2 \mathrm{AR}$ exposure may impede CFTR function in a cAMP-independent fashion. Notably, prior reports indicate that exposure to albuterol and $\beta 2 \mathrm{AR}$ internalization can increase CFTR expression (37). While we have not directly quantified membrane-bound CFTR, it is unlikely that this effect is implicit in our results, as this would predict the opposite effect - an increase in available CFTR and function. Additionally, Western blot results from Figure 5 suggest no change in mature C-band CFTR following chronic stimulation, and no significant change was noted in apical CFTR on qualitative immunofluorescence studies (Figure 6).

Our studies (with the exception of Figure 3 ) used a model of continuous exposure to albuterol to activate $\beta 2 \mathrm{AR}$ signaling. The dose of albuterol $(10 \mu \mathrm{M})$ has been described in pulmonary edema fluid from adult patients following therapeutic dosing; however, the kinetics of the drug at the epithelial surface over time are unclear (33). Our results (Figure 3) suggest that the negative effect of tonic albuterol exposure is recapitulated by both continuous and intermittent dosing with the LABA formoterol. While these results will require confirmation and demonstration in vivo, they highlight the potential vulnerability of corrected F508del-CFTR to this negative drug-drug interaction in primary HAECs.

The gene expression changes seen in our RNAseq studies following albuterol exposure were generally small, and there were no changes in the expression of any AC isoforms (which were expressed at low baseline levels) or in other members of the CFTR regulation pathway, with only minor ( $<2$-fold) increases in CFTR, MRP4, and one $\mathrm{G}_{\mathrm{s}} \alpha$ isoform observed. None of these changes would be predicted to produce deficits in CFTR activation following albuterol exposure. One of the most upregulated genes in both $\mathrm{CF}$ and non-CF donors was MUC5AC, 1 of 2 primary airway mucins. Mucus metaplasia has been previously reported in allergic inflammation-primed animal models following chronic $\beta 2 \mathrm{AR}$-agonist exposure, but to our knowledge has not been identified in a nonsensitized human cell model (55-57). The relevance of these findings is unknown, but we speculate that CFTR defects and mucin changes may have contributed to the 


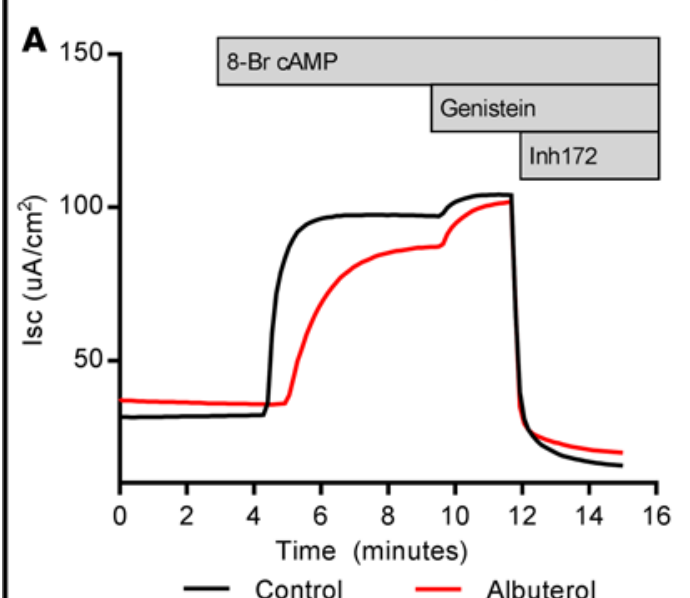

B

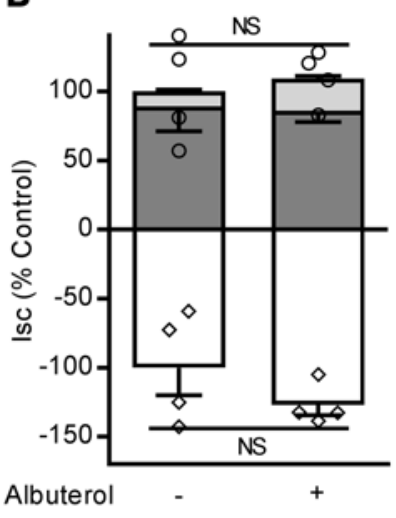

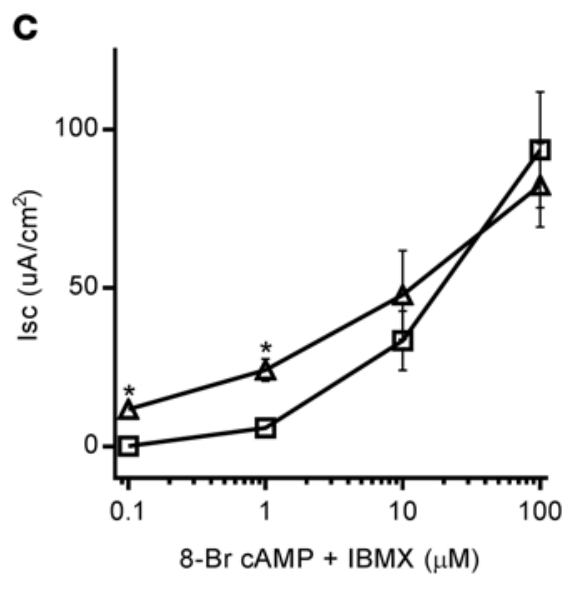

D

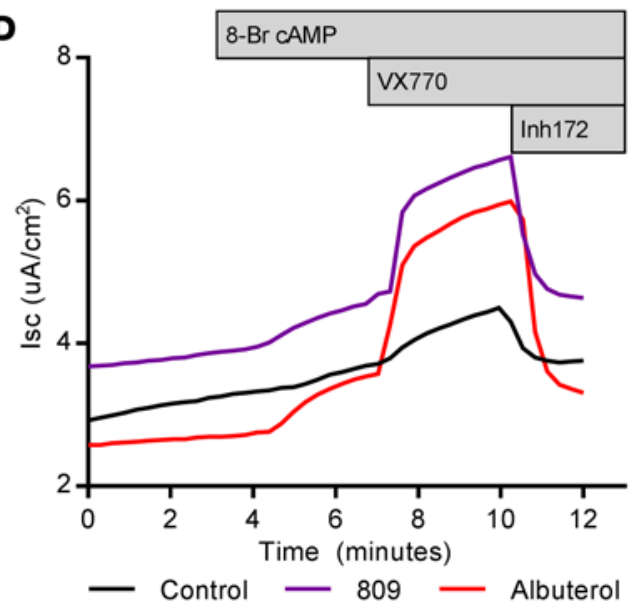

$\mathbf{E}$

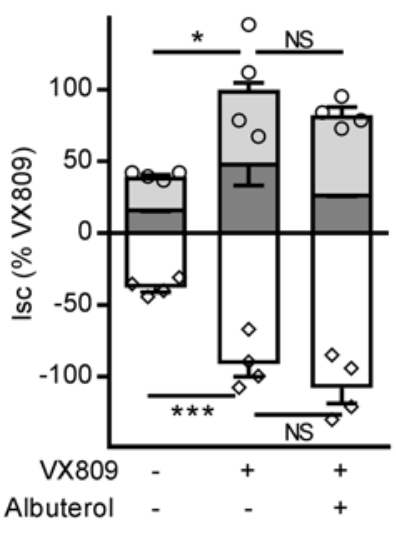

$\mathbf{F}$

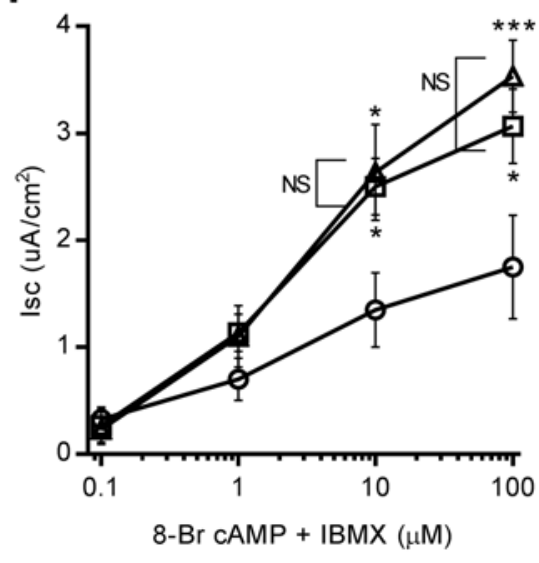

Figure 8. Chronic albuterol-induced CFTR impairment is rescued with application of exogenous CAMP. wtCFTR ${ }^{+}$and VX809-corrected F508del-CFTR ${ }^{+}$ CFBE410- cells were exposed to albuterol and/or VX809 in media for 72 hours, then mounted in Ussing chambers and CFTR function was assessed under voltage clamp conditions. In contrast to previously demonstrated experiments, cell-permeant 8-bromoadenosine cAMP (8-Br cAMP) was used to stimulate CFTR function to bypass cellular mechanisms of cAMP generation. In wtCFTR+ ${ }^{+}$cells (representative short-circuit current [I ${ }_{s c}$ tracing in $\mathbf{A}$, aggregate data in B; $n=4$ inserts/condition. Circles represent total CFTR activity [cAMP + genistein], diamonds represent inhibited CFTR currents [Inh172]), maximal stimulation with 8-Br CAMP in place of forskolin/IBMX rescues the previously demonstrated albuterol-induced CFTR dysfunction. Stimulation with increasing doses of 8-Br CAMP (C, $n=3$ inserts/data point) revealed a small increase in CFTR function in albuterol-pretreated cells at low stimulation doses. Squares, control conditions; triangles, albuterol pretreated. Similarly, in VX809-corrected F508del-CFTR ${ }^{+}$cells (representative $\mathrm{I}_{\mathrm{sc}}$ tracing in $\mathbf{D}$, aggregate data in E; $n=4$ inserts/condition), maximal stimulation with 8-Br cAMP rescues albuterol-induced CFTR dysfunction. Unlike wtCFTR ${ }^{+}$cells, stimulation with increasing doses of 8-Br cAMP demonstrated no difference in VX809-corrected F508del-CFTR function following albuterol pretreatment at any stimulation dose ( $F, n=3$ inserts/data point. All indicators of statistical significance are comparisons with untreated cells; no difference was noted between VX809-corrected cells with or without albuterol). Circles, untreated; squares, VX809 alone; triangles, VX809 + albuterol. All studies are internally normalized as indicated to allow for comparisons. Stimulation protocol was as follows: amiloride (100 $\mu \mathrm{M}$, not shown), cAMP (100 $\mu \mathrm{M} 8-\mathrm{Br}$ cAMP; dark gray bars), CFTR potentiator ( $50 \mu \mathrm{M}$ genistein for wtCFTR $; 1 \mu \mathrm{M}$ VX770 for F508del-CFTR ${ }^{+}$cells; light gray bars), and CFTR inhibition (10 $\mu \mathrm{M}$ Inh172; white bars). Both experiments are representative of studies repeated in duplicate with similar results. Data presented represent the mean \pm SEM. ${ }^{*} P<0.05$; ${ }^{* * *} P<$ 0.005 ; NS, non-significant by 2-way ANOVA with Tukey's multiple comparisons test.

reduction in MCC (Figure 4), and may further contribute to suboptimal patient responses to modulator therapy. Further studies to better understand the cellular and protein-level changes downstream of these transcriptional effects will be necessary to confirm whether they are of relevance to clinical care.

There are a number of limitations to the present results that should be considered. First, we have evaluated only cultures of epithelial cells. While the airway epithelium is typically considered the primary source of respiratory pathology in $\mathrm{CF}$, the typical effector tissue for $\beta 2 \mathrm{AR}$-agonists is airway smooth muscle. Recent studies have demonstrated clear evidence of smooth muscle dysfunction in $\mathrm{CF}$, further raising the importance of understanding the relative impact of $\beta 2 \mathrm{AR}$ stimulation on epithelial versus smooth muscle function in the context of defective and restored CFTR function (58). While our data clearly demonstrate 
the negative impact of chronic $\beta 2 \mathrm{AR}$-agonist exposure on subsequent CFTR activation, they do not fully evaluate possible modifications to the CFTR, such as changes in channel-open probability, plasma membrane recycling rate, or single-channel conductance. Surface biotinylation studies would directly evaluate effects on the apical membrane CFTR pool; however, such studies can be biased by permeable cells (59). The capacity to overcome functional defects with cell-permeant cAMP suggests that these are not operative, but our studies cannot exclude their contribution. Finally, the metabolism and breakdown of albuterol in our cell culture system is likely quite different from that in an intact human airway. Moreover, the cellular response to such exposures may be different in a living, complex organism; as such, the effects of our in vitro studies must be taken with caution when considering the in vivo condition.

In summary, our results are the first to demonstrate clear reduction in wtCFTR and corrected F508delCFTR activation following chronic exposure to $\beta 2 \mathrm{AR}$-agonists. This raises a critical clinical question in $\mathrm{CF}$ care, as excessive use of $\beta 2 \mathrm{AR}$-agonist therapies may inadvertently reduce the benefit from novel, potentially life-changing CFTR modulator therapies. This effect may also confound ongoing and future clinical trials of CFTR-modulating drugs, complicating interpretation of study results. Moreover, our results may have implications for other airway disease states where CFTR function is reduced (COPD), or where $\beta 2 \mathrm{AR}$-agonist use may be excessive (difficult-to-treat asthma). Further understanding the interplay between $\beta 2 \mathrm{AR}$-agonists and regulation of airway epithelial ion transport and CFTR will be essential to ensure maximal benefits from CFTR-directed therapies.

\section{Methods}

Cell culture. CFBE41o- cells were originally generated and described by Guenert and colleagues $(60,61)$. CFBE41o- cells stably transduced with wtCFTR or F508del-CFTR cDNA under regulatory control were the gift of Eric Sorscher at the University of Alabama at Birmingham (62). Cells were grown in T75 flasks (Costar), seeded onto collagen-coated Transwell filters (6.5 mm; Corning, Inc.), and maintained in DMEM containing 10\% FBS (Life Technologies). Resistance was monitored using an EVOM ${ }^{2}$ (World Precision Instruments), and cells were studied when resistance was 500-1,000 $\Omega \mathrm{cm}^{2}$. Primary HAECs obtained from the University of North Carolina Airway Cell Core (under the direction of Scott Randell; Chapel Hill, North Carolina) were isolated from donor or recipient lungs that were cryopreserved at passage 1 and cultured as previously described (63). Briefly, HAECs were cultured in Bronchial Epithelial Growth Medium (Lonza) on Purecol-coated tissue-culture dishes (Advanced Biomatrix) until 80\%-90\% confluent, then passaged and plated onto Transwell-Clear permeable supports $(0.4 \mu \mathrm{m}$ pore size; Corning, Inc.) coated with type IV collagen (Sigma-Aldrich). Cells on permeable supports were cultured with Ultroser G-containing (Crescent Chemical Company) media that was changed daily.

During the pretreatment period, all cells (CFBE41o- and primary HAEC) were changed to Ultroser Gcontaining media (in lieu of FBS) to minimize variability. To correct F508del-CFTR trafficking, F508del/ F508del AECs were treated with VX-809 (3 $\mu \mathrm{M}$; Selleck Chemicals) for 72 hours prior to study (38). For chronic exposure to cAMP-elevating agents, cells were treated for 72 hours prior to study with $0.083 \%$ albuterol (Nephron Pharmaceuticals Corporation - media changed daily) at the indicated concentrations. Routine mycoplasma testing (MTT kit; ATCC Bioproducts) was performed on all cell lines. Formoterol, MK571, and 8-bromoadenosine 3',5'-cyclic monophosphate were purchased from Sigma-Aldrich. GlyH101 was purchased from Millipore. Rolipram was purchased from Tocris Biosciences.

Ion transport. $\mathrm{I}_{\mathrm{sc}}$ and resistance were measured using Acquire and Analyze 2.3 software (Physiologic Instruments) as previously described (64). CFBE41o- cells and primary HAECs were placed in Ussing chambers and allowed to equilibrate for 30 minutes in symmetric $\mathrm{Cl}^{-}$buffers prior to study. Subsequently, cells were placed under voltage clamp conditions followed by a change to a $6 \mathrm{mM} \mathrm{Cl}^{-}$apical buffer (gluconate replaced $\mathrm{Cl}^{-}$) to produce a basolateral to apical $\mathrm{Cl}^{-}$secretory gradient. Cells were then treated with $100 \mu \mathrm{M}$ amiloride (apical compartment; Spectrum Chemical Corporation) to block epithelial sodium channel and $\mathrm{Na}^{+}$transport. CFTR was activated with apical/basolateral forskolin (10 $\mu \mathrm{M}$; Sigma-Aldrich) and IBMX (100 $\mu \mathrm{M}$; Acros Organics) and then potentiated with apical genistein (50 $\mu \mathrm{M}$, Sigma-Aldrich, wtCFTR) or VX770 (1 $\mu$ M, Selleck Chemicals, F508del/F508del). Forksolin/IBMX was selected to stimulate CFTR, bypassing the $32 \mathrm{AR}-\mathrm{GP}$ and directly stimulating AC activity. For permeabilization studies, 50 $\mu \mathrm{g} / \mathrm{ml}$ nystatin (Sigma-Aldrich) was added to the basolateral membrane as previously described (65). At the end of all studies, CFTRinh172 (10 $\mu \mathrm{M}$; Tocris Biosciences) was added to the apical compartment to block CFTR currents. 
To allow for comparisons between experiments, all studies were internally normalized to the average control (wtCFTR ${ }^{+}$) or VX809-corrected $\left(\right.$F508del-CFTR ${ }^{+}$) $\mathrm{I}_{\mathrm{sc}}$ and reported as percentage of the internal control condition.

Protein isolation. Cells on inserts were exposed to $150 \mu \mathrm{l}$ of Complete Lysis-M Buffer (Roche Diagnostics) for approximately 10 minutes, then manually scraped using a $200-\mu 1$ pipette tip. The resultant lysate was centrifuged at $300 \mathrm{~g}$ for 5 minutes and the supernatant was separated from the cell pellet. Protein quantification was performed using the DC Protein Assay (Bio-Rad). Immunoblotting was performed as previously described (64). Primary antibody against CFTR (mouse anti-CFTR clone 570; 1:4,000; University of North Carolina at Chapel Hill) was added to the blots and incubated overnight at $4^{\circ} \mathrm{C}$, then horseradish peroxidase-conjugated secondary antibody (goat anti-mouse 1:200,000; Southern Biotech, catalog 103005) was applied in TBS-TII (10 mM Tris-HCl, $500 \mathrm{mM} \mathrm{NaCl}, 0.5 \%$ Tween-20, pH 7.4). Immunoreactivity was detected by chemiluminescence (SuperSignal West Femto, Thermo Fisher Scientific) and quantified using Image J software (NIH).

Immunofluorescence and colocalization analysis. Mature cells on inserts were washed 3 times in $1 \times$ PBS for 5 minutes, then fixed in 4\% paraformaldehyde (Electron Microscopy Sciences) overnight. 1\% Triton X-100 (Sigma-Aldrich) in PBS was applied for 15 minutes to permeabilize cells at room temperature, followed by blocking with $2.5 \%$ horse serum (Vector) for 3 hours. Cells were again washed 3 times in $1 \times$ PBS. Immunostaining was then performed using an overnight $4^{\circ} \mathrm{C}$ incubation for primary antibodies and 1-hour room temperature incubation for secondary antibodies; all antibody concentrations and catalog numbers are documented in Supplemental Table 1. Membranes and cells were washed 3 times in $1 \times$ PBS and then cut away from the plastic insert with a razor and mounted onto slides with ProLong Gold Antifade with DAPI (Thermo Fisher Scientific).

Imaging was performed with a Nikon A1 inverted confocal microscope and captured using Nikon Elements software with equivalent microscope and camera settings for each paired image (control vs. albuterol). Analysis of colocalization of $\beta 2 \mathrm{AR}$ and CFTR was performed using Imaris (Bitplane) software. Three separate image sets, each from a separate slide/insert, were analyzed per condition. Colocalization analysis included both single images in the $x-y$ and $x-z$ planes (shown) and 3-dimensional reconstruction images in the $x-y-z$ plane. Colocalization was quantified as the percentage of colocalized pixels (positive for both green and purple channels) above a common threshold. For each paired set of images, thresholds were set to analyze the top $40 \%$ of fluorescent signal in the control images; the same thresholds were applied to analysis of the paired albuterol-treated images to ensure equivalent analyses within subjects while minimizing noise. Colocalization was calculated both for the entire image and for a manually delineated region of interest (ROI) focused on the apical surface only.

cAMP measurements. wtCFTR ${ }^{+}$and F508del-CFTR ${ }^{+}$CFBE41o- cells were pretreated for 72 hours with albuterol $(10 \mu \mathrm{M})$. cAMP levels were measured following a 30-minute washout (media only). As indicated in the Results, select cells were stimulated with forskolin/IBMX (10 $\mu \mathrm{M} / 100 \mu \mathrm{M}$ in HBSS) or vehicle for 10 minutes. Cells were then lysed with a solution of $0.1 \mathrm{M} \mathrm{HCl}$ and $0.1 \%$ Triton and centrifuged. cAMP levels in the supernatant were then immediately analyzed by colorimetric ELISA (Enzo Life Sciences) per the manufacturer's recommended protocol. cAMP values were calculated and normalized to the stimulated average to allow for comparison.

Ciliary motility and MCC. Mature, $\mathrm{wCFTR}^{+}$primary HAECs were grown as described above to maturity. Seventy-two hours prior to study, $50 \mu \mathrm{l}$ of PBS was applied to wash the apical surface of each insert for 10 minutes, then removed. Fluorescent beads (1:500 dilution in PBS; Polysciences, Inc.) were then applied to the apical surface in aliquots of $100 \mu 1$ per insert and left in place until study, allowing airway surface liquid levels to return to equilibrium. Inserts were placed right-side up onto a drop of media in a $35-\mathrm{mm}$ glass-bottom dish (MatTek Corporation) and mounted in an incubated chamber at $37^{\circ} \mathrm{C}$ and $5 \% \mathrm{CO}_{2}$ on a Nikon TiE inverted microscope with Andor Zyla 4.2 and a Lumencor Spectra X light source.

For cilia imaging, high-speed time-lapse microscopy (approximately 400 frames/second) was performed at $\times 40$ magnification under DIC conditions. All images were captured within 1 field of the insert edge to limit variability. Ciliary beat frequency was analyzed using Nikon Elements software (Nikon Instruments)

For MCC, time-lapse microscopy (approximately 20 frames/second) was performed at $\times 20$ magnification and $640 \mathrm{~nm}$. All images were captured within 1 field of the insert edge. Individual beads were marked and bead movement speed was calculated using Imaris software. 
RNA isolation, sequencing, and analysis. Cells on inserts were lysed and RNA was collected using the RNeasy Mini Kit (Qiagen) and the manufacturer's supplied protocol. Quality control testing was performed using a Nanodrop 2000c (Thermo Fisher Scientific) and Bioanalyzer RNA kit (Agilent Technologies). RNA was amplified and converted to cDNA using the Ovation RNA-seq System v2 (NuGEN Technologies) and cDNA libraries were created using the Illumina Protocol and Nextera XT DNA Library Preparation Kit (Illumina). Sequencing was then performed with the aid of the Cincinnati Children's Hospital Research Foundation DNA Sequencing Core, with 25-30 million reads using paired-end 75-bp flow cells. Kallisto was used for quantifying abundance of transcripts from RNAseq data (66). The differentially expressed genes were detected using the R package DESeq2 (67). An a priori-adjusted significance value of 0.01 was used to determine statistical change.

Statistics. All comparisons of continuous data (including $\mathrm{I}_{\mathrm{sc}}$, densitometry, cAMP levels, MCC, and ciliary beat frequency) were made by 2-sided Student's $t$ test or 1- or 2-way ANOVA as appropriate, with appropriate correction for multiple comparisons, using Graphpad Prism software. An a priori significance $(P)$ value of 0.05 was used for all comparisons. Data presented throughout represent the mean \pm SEM unless stated otherwise. Unless otherwise stated, all studies presented were repeated in duplicate or triplicate, with similar results.

Study approval. All studies using primary HAECs were approved by the Cincinnati Children's Hospital Medical Center Institutional Review Board.

\section{Author contributions}

$\mathrm{JJB}, \mathrm{JB}, \mathrm{AF}, \mathrm{EK}, \mathrm{JM}$, and AO directly contributed to study design, conducting experiments, data analysis, and the intellectual content of the manuscript. LJL and XZ contributed to data analysis and manuscript revision. JPC contributed to study design, data analysis, and the intellectual content of the manuscript.

\section{Acknowledgments}

This work was supported by funding from the Cystic Fibrosis Foundation (CLANCY15R0 and BREWIN16A0). X. Zhu is supported by National Institutes of Health Heart Lung and Blood Institute award HL111829 to L.J. Lu.

The authors wish to thank Anjaparavanda Naren and Satish Madala for their advice and support throughout the course of this work.

Address correspondence to: John Brewington, 3333 Burnet Avenue, MLC 2021, Cincinnati, Ohio 45229, USA. Phone: 513.636.6771; Email: john.brewington@cchmc.org.

1. Welsh MJ. An apical-membrane chloride channel in human tracheal epithelium. Science. 1986;232(4758):1648-1650.

2. Rowe SM, Miller S, Sorscher EJ. Cystic fibrosis. N Engl J Med. 2005;352(19):1992-2001.

3. CFTR2 Database. The Clinical and Functional TRanslation of CFTR (CFTR2) http://www.cftr2.org. Accessed January $29,2018$.

4. Brewington JJ, McPhail GL, Clancy JP. Lumacaftor alone and combined with ivacaftor: preclinical and clinical trial experience of F508del CFTR correction. Expert Rev Respir Med. 2016;10(1):5-17.

5. Pettit RS, Fellner C. CFTR modulators for the treatment of cystic fibrosis. P T. 2014;39(7):500-511.

6. Wainwright CE, et al. Lumacaftor-ivacaftor in patients with cystic fibrosis homozygous for Phe508del CFTR. N Engl JMed. 2015;373(3):220-231.

7. Ramsey BW, et al. A CFTR potentiator in patients with cystic fibrosis and the G551D mutation. N Engl J Med. 2011;365(18):1663-1672.

8. Moss RB, et al. Efficacy and safety of ivacaftor in patients with cystic fibrosis who have an Arg117His-CFTR mutation: a double-blind, randomised controlled trial. Lancet Respir Med. 2015;3(7):524-533.

9. Boucher RC. Human airway ion transport. Part one. Am J Respir Crit Care Med. 1994;150(1):271-281.

10. Boucher RC. Evidence for airway surface dehydration as the initiating event in CF airway disease. J Intern Med. 2007;261(1):5-16.

11. Riordan JR. The cystic fibrosis transmembrane conductance regulator. Annu Rev Physiol. 1993;55:609-630.

12. Pasyk EA, et al. A conserved region of the $\mathrm{R}$ domain of cystic fibrosis transmembrane conductance regulator is important in processing and function. J Biol Chem. 1998;273(48):31759-31764.

13. Anderson MP, Berger HA, Rich DP, Gregory RJ, Smith AE, Welsh MJ. Nucleoside triphosphates are required to open the CFTR chloride channel. Cell. 1991;67(4):775-784.

14. Lee JH, et al. Dynamic regulation of cystic fibrosis transmembrane conductance regulator by competitive interactions of molecular adaptors. J Biol Chem. 2007;282(14):10414-10422.

15. Naren AP, et al. A macromolecular complex of beta 2 adrenergic receptor, CFTR, and ezrin/radixin/moesin-binding phosphoprotein 50 is regulated by PKA. Proc Natl Acad Sci USA. 2003;100(1):342-346. 
16. Limbird LE, Gill DM, Lefkowitz RJ. Agonist-promoted coupling of the beta-adrenergic receptor with the guanine nucleotide regulatory protein of the adenylate cyclase system. Proc Natl Acad Sci USA. 1980;77(2):775-779.

17. Hepler JR, Gilman AG. G proteins. Trends Biochem Sci. 1992;17(10):383-387.

18. Li C, Naren AP. CFTR chloride channel in the apical compartments: spatiotemporal coupling to its interacting partners. Integr Biol (Camb). 2010;2(4):161-177.

19. Field M, Fromm D, al-Awqati Q, Greenough WB. Effect of cholera enterotoxin on ion transport across isolated ileal mucosa. J Clin Invest. 1972;51(4):796-804.

20. Gabriel SE, Brigman KN, Koller BH, Boucher RC, Stutts MJ. Cystic fibrosis heterozygote resistance to cholera toxin in the cystic fibrosis mouse model. Science. 1994;266(5182):107-109.

21. Hanoune J, Defer N. Regulation and role of adenylyl cyclase isoforms. Annu Rev Pharmacol Toxicol. 2001;41:145-174.

22. Zhang G, Liu Y, Ruoho AE, Hurley JH. Structure of the adenylyl cyclase catalytic core. Nature. 1997;386(6622):247-253.

23. Mogayzel PJ, et al. Cystic fibrosis pulmonary guidelines. Chronic medications for maintenance of lung health. Am J Respir Crit Care Med. 2013;187(7):680-689.

24. 2014 CFF Patient Registry Annual Data Report. Cystic Fibrosis Foundation. https://www.cff.org/2014-Annual-Data-Report. pdf. Accessed January 29, 2018.

25. Sabater JR, Lee TA, Abraham WM. Comparative effects of salmeterol, albuterol, and ipratropium on normal and impaired mucociliary function in sheep. Chest. 2005;128(5):3743-3749.

26. Eggleston PA, Rosenstein BJ, Stackhouse CM, Mellits ED, Baumgardner RA. A controlled trial of long-term bronchodilator therapy in cystic fibrosis. Chest. 1991;99(5):1088-1092.

27. König P, Poehler J, Barbero GJ. A placebo-controlled, double-blind trial of the long-term effects of albuterol administration in patients with cystic fibrosis. Pediatr Pulmonol. 1998;25(1):32-36.

28. Chen Y, et al. Adenylyl cyclase 6 is selectively regulated by protein kinase A phosphorylation in a region involved in Galphas stimulation. Proc Natl Acad Sci USA. 1997;94(25):14100-14104.

29. Iwami G, Kawabe J, Ebina T, Cannon PJ, Homcy CJ, Ishikawa Y. Regulation of adenylyl cyclase by protein kinase A. J Biol Chem. 1995;270(21):12481-12484.

30. Lohse MJ, Benovic JL, Caron MG, Lefkowitz RJ. Multiple pathways of rapid beta 2-adrenergic receptor desensitization. Delineation with specific inhibitors. J Biol Chem. 1990;265(6):3202-3211.

31. Benovic JL, Staniszewski C, Mayor F, Caron MG, Lefkowitz RJ. beta-Adrenergic receptor kinase. Activity of partial agonists for stimulation of adenylate cyclase correlates with ability to promote receptor phosphorylation. J Biol Chem. 1988;263(8):3893-3897.

32. Sun $\mathrm{H}$, et al. Tgf-beta downregulation of distinct chloride channels in cystic fibrosis-affected epithelia. PLoS One. 2014;9(9):e106842.

33. Atabai K, et al. Aerosolized beta(2)-adrenergic agonists achieve therapeutic levels in the pulmonary edema fluid of ventilated patients with acute respiratory failure. Intensive Care Med. 2002;28(6):705-711.

34. Barnes AP, et al. Phosphodiesterase 4D forms a cAMP diffusion barrier at the apical membrane of the airway epithelium. $J$ Biol Chem. 2005;280(9):7997-8003.

35. Blanchard E, et al. Anchored PDE4 regulates chloride conductance in wild-type and $\triangle$ F508-CFTR human airway epithelia. FASEB J. 2014;28(2):791-801.

36. Conner GE, Ivonnet P, Gelin M, Whitney P, Salathe M. H2O2 stimulates cystic fibrosis transmembrane conductance regulator through an autocrine prostaglandin pathway, using multidrug-resistant protein-4. Am J Respir Cell Mol Biol. 2013;49(4):672-679.

37. Taouil K, Hinnrasky J, Hologne C, Corlieu P, Klossek JM, Puchelle E. Stimulation of beta 2-adrenergic receptor increases cystic fibrosis transmembrane conductance regulator expression in human airway epithelial cells through a cAMP/protein kinase A-independent pathway. J Biol Chem. 2003;278(19):17320-17327.

38. Van Goor F, et al. Correction of the F508del-CFTR protein processing defect in vitro by the investigational drug VX-809. Proc Natl Acad Sci USA. 2011;108(46):18843-18848.

39. Rowe SM, et al. Tezacaftor-ivacaftor in residual-function heterozygotes with cystic fibrosis. N Engl J Med. 2017;377(21):2024-2035.

40. Taylor-Cousar JL, et al. Tezacaftor-ivacaftor in patients with cystic fibrosis homozygous for Phe508del. N Engl J Med. 2017;377(21):2013-2023.

41. Kerem E, et al. Ataluren for the treatment of nonsense-mutation cystic fibrosis: a randomised, double-blind, placebo-controlled phase 3 trial. Lancet Respir Med. 2014;2(7):539-547.

42. Nick JA, et al. Azithromycin may antagonize inhaled tobramycin when targeting Pseudomonas aeruginosa in cystic fibrosis. Ann Am Thorac Soc. 2014;11(3):342-350.

43. Wang WC, Schillinger RM, Malone MM, Liggett SB. Paradoxical attenuation of $\beta 2$-AR function in airway smooth muscle by Gi-mediated counterregulation in transgenic mice overexpressing type 5 adenylyl cyclase. Am J Physiol Lung Cell Mol Physiol. 2011;300(3):L472-L478.

44. Penn RB, Kelsen SG, Benovic JL. Regulation of beta-agonist- and prostaglandin E2-mediated adenylyl cyclase activity in human airway epithelial cells. Am J Respir Cell Mol Biol. 1994;11(4):496-505.

45. Wang WC, et al. Targeted transgenesis identifies Gas as the bottleneck in $\beta 2$-adrenergic receptor cell signaling and physiological function in airway smooth muscle. Am J Physiol Lung Cell Mol Physiol. 2014;307(10):L775-L780.

46. Moon C, et al. Compartmentalized accumulation of cAMP near complexes of multidrug resistance protein 4 (MRP4) and cystic fibrosis transmembrane conductance regulator (CFTR) contributes to drug-induced diarrhea. J Biol Chem. 2015;290(18):11246-11257.

47. Sellers ZM, Naren AP, Xiang Y, Best PM. MRP4 and CFTR in the regulation of cAMP and $\beta$-adrenergic contraction in cardiac myocytes. Eur J Pharmacol. 2012;681(1-3):80-87.

48. Kramer E, Backstrom J, Ostmann AJ, Sun H, Naren AP, Clancy JP. Negative effects of prolonged beta agonist and cAMP treatment on wt and VX-809 corrected F508del CFTR currents (but not CFTR maturation) in human airway cells. Pediatr Pulmonol. 2014;49(S38):257

49. Post SR, Hilal-Dandan R, Urasawa K, Brunton LL, Insel PA. Quantification of signalling components and amplification in the beta-adrenergic-receptor-adenylate cyclase pathway in isolated adult rat ventricular myocytes. Biochem J. 1995;311(Pt 1):75-80 
50. Alousi AA, Jasper JR, Insel PA, Motulsky HJ. Stoichiometry of receptor-Gs-adenylate cyclase interactions. FASEB J. 1991;5(9):2300-2303.

51. Schreiber R, Kindle P, Benzing T, Walz G, Kunzelmann K. Control of the cystic fibrosis transmembrane conductance regulator by alphaG(i) and RGS proteins. Biochem Biophys Res Commun. 2001;281(4):917-923.

52. Loffing J, Moyer BD, McCoy D, Stanton BA. Exocytosis is not involved in activation of Cl- secretion via CFTR in Calu-3 airway epithelial cells. Am J Physiol. 1998;275(4 Pt 1):C913-C920.

53. Hug MJ, Thiele IE, Greger R. The role of exocytosis in the activation of the chloride conductance in Chinese hamster ovary cells (CHO) stably expressing CFTR. Pflugers Arch. 1997;434(6):779-784.

54. Weber WM, Cuppens H, Cassiman JJ, Clauss W, Van Driessche W. Capacitance measurements reveal different pathways for the activation of CFTR. Pflugers Arch. 1999;438(4):561-569.

55. Kamachi A, et al. Enhancement of goblet cell hyperplasia and airway hyperresponsiveness by salbutamol in a rat model of atopic asthma. Thorax. 2001;56(1):19-24.

56. Nguyen LP, et al. Beta2-adrenoceptor signaling is required for the development of an asthma phenotype in a murine model. Proc Natl Acad Sci USA. 2009;106(7):2435-2440.

57. Lin $\mathrm{R}$, et al. Chronic treatment in vivo with $\beta$-adrenoceptor agonists induces dysfunction of airway $\beta(2)$-adrenoceptors and exacerbates lung inflammation in mice. Br J Pharmacol. 2012;165(7):2365-2377.

58. Cook DP, et al. Cystic fibrosis transmembrane conductance regulator in sarcoplasmic reticulum of airway smooth muscle. Implications for airway contractility. Am J Respir Crit Care Med. 2016;193(4):417-426.

59. Luo Y, McDonald K, Hanrahan JW. Trafficking of immature DeltaF508-CFTR to the plasma membrane and its detection by biotinylation. Biochem J. 2009;419(1):211-219.

60. Haws C, Krouse ME, Xia Y, Gruenert DC, Wine JJ. CFTR channels in immortalized human airway cells. Am J Physiol. 1992;263(6 Pt 1):L692-L707.

61. Meng QH, et al. Lack of inducible nitric oxide synthase in bronchial epithelium: a possible mechanism of susceptibility to infection in cystic fibrosis. J Pathol. 1998;184(3):323-331.

62. Bebok Z, et al. Failure of cAMP agonists to activate rescued deltaF508 CFTR in CFBE41o- airway epithelial monolayers. J Physiol (Lond). 2005;569(Pt 2):601-615.

63. Fulcher ML, Gabriel S, Burns KA, Yankaskas JR, Randell SH. Well-differentiated human airway epithelial cell cultures. Methods Mol Med. 2005;107:183-206.

64. Rowe SM, et al. DeltaF508 CFTR processing correction and activity in polarized airway and non-airway cell monolayers. Pulm Pharmacol Ther. 2010;23(4):268-278.

65. Ito Y, Sato S, Ohashi T, Nakayama S, Shimokata K, Kume H. Reduction of airway anion secretion via CFTR in sphingomyelin pathway. Biochem Biophys Res Commun. 2004;324(2):901-908.

66. Bray NL, Pimentel H, Melsted P, Pachter L. Near-optimal probabilistic RNA-seq quantification. Nat Biotechnol. 2016;34(5):525-527.

67. Love MI, Huber W, Anders S. Moderated estimation of fold change and dispersion for RNA-seq data with DESeq2. Genome Biol. 2014;15(12):550. 